\title{
Parafermionic character formulae
}

\author{
P. Jacob and P. Mathieu \\ Département de physique, \\ Université Laval, \\ Québec, Canada G1K 7P4 \\ (pjacob@phy.ulaval.ca, pmathieu@phy.ulaval.ca)
}

\begin{abstract}
We study various aspects of parafermionic theories such as the precise field content, a description of a basis of states (that is, the counting of independent states in a freely generated highest-weight module) and the explicit expression of the parafermionic singular vectors in completely irreducible modules. This analysis culminates in the presentation of new character formulae for the $Z_{N}$ parafermionic primary fields. These characters provide novel field theoretical expressions for $\widehat{s u}(2)$ string functions.
\end{abstract}

$05 / 00$

1 Work supported by NSERC (Canada) and FCAR (Québec) 


\section{Introduction}

The free-fermion description of the Ising model is a crucial ingredient in our rather elaborate understanding of its transition from the conformal point to its off-critical version. This property definitely distinguishes it from the other minimal models. In fact, this description of the Ising model makes rather direct the contact with its statistical formulation. The Ising model is also singled out in that this is the only case for which the correlation functions have been proved to satisfy (integrable) differential equations which reduce, at criticality, to the precise differential equations that follow from the structure of the singular vectors (see for instance [1]).

The most natural way of extending these results is probably to look for conformal theories whose formulation conserves much of the structure of their statistical relative and which generalizes the Ising model in the most natural way. That singles out the three-state Potts model and, more generally, the parafermionic theories [2].

A very simple question that one can ask as a sort of probe of the smoothness of the transition from the Ising model to generic parafermionic theories is related to character formulae. The free-fermionic description of the Ising model leads to simple character expressions. These are characters in a free-fermionic Fock space, and these have a simple decomposition into characters of irreducible Virasoro modules:

$$
\begin{aligned}
& \chi_{1,1}=\frac{q^{-1 / 48}}{2}\left[\prod_{n=1}^{\infty}\left(1+q^{n-1 / 2}\right)+\prod_{n=1}^{\infty}\left(1-q^{n-1 / 2}\right)\right] \\
& \chi_{2,1}=\frac{q^{-1 / 48}}{2}\left[\prod_{n=1}^{\infty}\left(1+q^{n-1 / 2}\right)-\prod_{n=1}^{\infty}\left(1-q^{n-1 / 2}\right)\right] \\
& \chi_{1,2}=q^{1 / 24} \prod_{n=1}^{\infty}\left(1+q^{n}\right)
\end{aligned}
$$

However, a crucial aspect of the simplicity of the Ising free-field representation lies in the fact that this particular representation captures the whole set of singular vectors. It is faithful in that sense. In other words, there is no singular-vector subtraction. On the other hand, we do not expect the parafermionic description to be faithful.

But in order to construct the character directly from the parafermionic algebra, some aspects of the parafermionic representation theory are needed. 
In contradistinction to the situation with most extended conformal algebras, the representation theory of the parafermionic algebra has not been studied in much detail. Indeed, apart from the initial fundamental paper of Zamolodchikov and Fateev [2], which was followed by an extension dealing mainly with the relation to the $N=2$ supersymmetric theories [3], and a study of a special example of a more general class of models [4], most subsequent articles deal with the parafermionic theory either via a free-field representation (in particular, singular vectors and character formulae have been worked out in [5] from BRST methods using a representation introduced in [6] in terms of two free bosons - see also [7]) or use a correspondence with either WZW models (cf. the classification of modular invariants in [8]) or special minimal models of $\mathrm{W}$ algebras (e.g., cf. the analysis of the integrable perturbations in [9]).

This situation is manifestly due to the rather complicated structure of the algebra, whose mode formulation involves infinite sums.

Among the basic features that have never been worked out directly from the parafermionic algebra and which are needed in order to work out the character formulae, there are the counting of the number of independent states (i.e., a description of a basis of states) for a generic parafermionic highest-weight module, the explicit form of the singular vectors associated to the spin fields (the parafermionic primary fields) and the general structure of the corresponding irreducible modules.

These precise aspects of the parafermionic models are addressed here. We start by clarifying the field content (that is, the analogue of the Kac table for the Virasoro minimal models) of a given model, paying due care to the question of field identifications and compare it with that predicted by the simplest coset constructions. We then work out the explicit form of the spin-field singular vectors. These singular vectors are charged and it is shown how to generate descendents of appropriate relative charge. For particular cases (e.g. the Ising and the Potts models), the singular-vector descendents of zero relative charge can all be described in terms of the Virasoro modes and the familiar Virasoro singular-vector expressions are recovered; this is shown in appendix B. Finally, we present the parafermionic character formulae for the charged and the uncharged modules built on the highest-weight states corresponding to the spin fields.

The development of the representation theory of the parafermionic conformal field theory, which will always refer here to the basic models of [2], has interesting offshots. 
Indeed, many extensions of this class of models can be constructed and some (if not most) of which may not have a coset construction nor any simple relation to other extended conformal field theories. Relying upon genuine parafermionic constructions would then seems to be the only available tool at hand.

\section{Parafermionic conformal field theories}

In this section, we review briefly the main results of [2] that will be needed in the sequel.

The parafermionic algebra is constructed out of $N$ parafermionic fields $\psi_{k}, k=$ $0,1, \cdots, N-1$ (with $\psi_{0}=I$ and $\psi_{k}^{\dagger}=\psi_{N-k}$ ) of holomorphic conformal dimension

$$
h_{\psi_{k}}=\frac{k(N-k)}{N}
$$

that satisfy the OPE:

$$
\begin{aligned}
\psi_{k}(z) \psi_{k^{\prime}}(w) & \sim c_{k, k^{\prime}}(z-w)^{-2 k k^{\prime} / N} \psi_{k+k^{\prime}}(w) \quad\left(k^{\prime} \neq N-k\right) \\
\psi_{k}(z) \psi_{k}^{\dagger}(w) & \sim(z-w)^{-2 k(N-k) / N}\left[I+(z-w)^{2} \frac{2 h_{\psi_{k}}}{c} T(w)\right] \\
T(z) \psi_{k}(w) & \sim \frac{h_{\psi_{k}} \psi_{k}(w)}{(z-w)^{2}}+\frac{\partial \psi_{k}(w)}{(z-w)} \\
T(z) T(w) & \sim \frac{c / 2}{(z-w)^{4}}+\frac{2 T(w)}{(z-w)^{2}}+\frac{\partial T(w)}{(z-w)}
\end{aligned}
$$

The associativity conditions fix the constant $c_{k, k^{\prime}}$ to be

$$
c_{k, k^{\prime}}^{2}=\frac{\Gamma\left(k+k^{\prime}+1\right) \Gamma(N-k+1) \Gamma\left(N-k^{\prime}+1\right)}{\Gamma(k+1) \Gamma\left(k^{\prime}+1\right) \Gamma\left(N-k-k^{\prime}+1\right) \Gamma(N+1)}
$$

and the central charge is linked to $N$ by

$$
c=\frac{2(N-1)}{(N+2)}
$$

The space of fields $\mathcal{F}$ can be decomposed into a direct sum of charged spaces $\mathcal{F}_{[q, \bar{q}]}$, where $q, \bar{q}$ represent the holomorphic and antiholomorphic charges, defined modulo $2 N$. The charge normalization is fixed by the charge assignment of the parafermionic fields: $\psi_{k} \in \mathcal{F}_{[2 k, 0]}$ and $\bar{\psi}_{k} \in \mathcal{F}_{[0,2 k]}$. 
Let us indicate how this space of fields can be organized in terms of the parafermionic algebra as defined by the above OPEs. For this, it is convenient to describe the action of the parafermionic fields in terms of their modes. However, the precise form of the mode expansion depends upon the field on which the parafermion acts upon. For instance, on a field $\phi_{[q, \bar{q}]}$, the mode expansion of $\psi_{1}$ and $\psi_{1}^{\dagger}$ is defined as

$$
\begin{aligned}
& \psi_{1}(z) \phi_{[q, \bar{q}]}(0)=\sum_{m=-\infty}^{\infty} z^{-q / N-m-1} A_{(1+q) / N+m} \phi_{[q, \bar{q}]}(0) \\
& \psi_{1}^{\dagger}(z) \phi_{[q, \bar{q}]}(0)=\sum_{m=-\infty}^{\infty} z^{q / N-m-1} A_{(1-q) / N+m}^{\dagger} \phi_{[q, \bar{q}]}(0)
\end{aligned}
$$

As usual, we read off the dimension of a mode directly from the negative of its index:

$$
\begin{aligned}
& h_{A_{(1+q) / N+m} \phi_{[q, \bar{q}]}}=-\frac{1+q}{N}-m+h_{\phi_{[q, \bar{q}]}} \\
& h_{A_{(1-q) / N+m}^{\dagger} \phi_{[q, \bar{q}]}}=-\frac{1-q}{N}-m+h_{\phi_{[q, \bar{q}]}}
\end{aligned}
$$

and the charge of a mode is that of its corresponding field:

$$
q_{A_{(1+q) / N+m}} \phi_{[q, \bar{q}]}=2+q, \quad q_{A_{(1-q) / N+m}^{\dagger} \phi_{[q, \bar{q}]}}=-2+q
$$

Now, it turns out that the action of $\psi_{1}$ and $\psi_{1}^{\dagger}$ suffices to generate the whole space of fields. Before we make this explicit, we display the mode commutation relation. The action of the modes $A_{u}$ or $A_{v}^{\dagger}$ on a field with given charge is fixed by the inverted version of $(2.5)$

$$
\begin{aligned}
& A_{(1+q) / N+m} \phi_{[q, \bar{q}]}(0)=\frac{1}{2 \pi i} \oint_{0} d z \psi_{1}(z) z^{q / N+m} \phi_{[q, \bar{q}]}(0) \\
& A_{(1-q) / N+m}^{\dagger} \phi_{[q, \bar{q}]}(0)=\frac{1}{2 \pi i} \oint_{0} d z \psi_{1}^{\dagger}(z) z^{-q / N+m} \phi_{[q, \bar{q}]}(0)
\end{aligned}
$$

The commutation relation between $A_{u}$ and $A_{v}^{\dagger}$ follows from the computation of the integral $[2]:$

$$
\frac{1}{(2 \pi i)^{2}} \oint_{0} d w \oint_{w} d z \psi_{1}(z) \psi_{1}^{\dagger}(w) z^{q / N+m} w^{-q / N+m+1}(z-w)^{-(N+2) / N} \phi_{[q, \bar{q}]}(0)
$$

and this yields 2

$$
\begin{aligned}
& \sum_{l=0}^{\infty} c_{l}\left[A_{-(1-q) / N+n-l-1} A_{(1-q) / N+m+l+1}^{\dagger}+A_{-(q+1) / N+m-l}^{\dagger} A_{(1+q) / N+n+l}\right] \phi_{[q, \bar{q}]}(0) \\
& =\left[\frac{(N+2)}{N} L_{n+m}+\frac{1}{2}\left(n+\frac{q}{N}\right)\left(n-1+\frac{q}{N}\right) \delta_{n+m, 0}\right] \phi_{[q, \bar{q}]}(0)
\end{aligned}
$$

2 This are two misprints in [2] with regard to this expression: a power of $w$ is missing in the previous integral and the relative sign in the following commutation relation is incorrect. 
where

$$
c_{l}=C_{-(N+2) / N}^{(l)}, \quad C_{n}^{(p)}=\frac{\Gamma(p-n)}{p ! \Gamma(-n)}
$$

To get the commutation relation between $A_{u}$ and $A_{v}$, we need to evaluate

$$
\frac{1}{(2 \pi i)^{2}} \oint_{0} d w \oint_{w} d z \psi_{1}(z) \psi_{1}(w) z^{q / N+m} w^{q / N+m}(z-w)^{2 / N} \phi_{[q, \bar{q}]}(0)
$$

and that leads to

$$
\sum_{l=0}^{\infty} c_{l}^{\prime}\left[A_{(3+q) / N+n-l} A_{(1+q) / N+m+l}-A_{(3+q) / N+m-l} A_{(1+q) / N+n+l}\right] \phi_{[q, \bar{q}]}(0)=0
$$

where

$$
c_{l}^{\prime}=C_{2 / N}^{(l)}
$$

There is a similar expression with $A$ and $q$ replaced by $A^{\dagger}$ and $-q$. On the other hand, the commutation relations between the Virasoro and the parafermionic modes are

$$
\begin{aligned}
& {\left[L_{m}, A_{u}^{\dagger}\right]=-(u+m / N) A_{u+m}^{\dagger}} \\
& {\left[L_{m}, A_{v}\right]=-(v+m / N) A_{v+m}}
\end{aligned}
$$

The parafermionic primary fields are called the spin fields $\sigma_{k} \in \mathcal{F}_{[k, k]}, k=0,1, \cdots, N-$ 1. They are associated to the highest-weight states $\left|\sigma_{k}\right\rangle$ satisfying the holomorphic highestweight conditions

$$
A_{(1+k) / N+n}\left|\sigma_{k}\right\rangle=A_{(1-k) / N+n+1}^{\dagger}\left|\sigma_{k}\right\rangle=L_{n+1}\left|\sigma_{k}\right\rangle=0 \quad \text { for } \quad n \geq 0
$$

Actually, it follows from the commutation relation (2.10) with $n=m=0$ that there can exist only one primary field of a given charge and its conformal dimension is fixed directly by the algebra to be:

$$
h_{\sigma_{k}}=\frac{k(N-k)}{2 N(N+2)}
$$

Equivalently, the primary fields could be chosen to be the disorder fields $\mu_{k} \in \mathcal{F}_{[-k, k]}$; the corresponding states satisfy the holomorphic highest-weight conditions:

$$
A_{(1-k) / N+n+1}\left|\mu_{k}\right\rangle=A_{(1+k) / N+n}^{\dagger}\left|\mu_{k}\right\rangle=L_{n+1}\left|\mu_{k}\right\rangle=0 \quad \text { for } \quad n \geq 0
$$

and it follows from (2.10) with $n=-m=1$ that $h_{\mu_{k}}=h_{\sigma_{k}}$. Notice that with this definition for the disorder field, we have $\sigma_{k} \mu_{-k} \sim \psi_{k}$ and $\sigma_{k} \mu_{k} \sim \bar{\psi}_{k}$. 
The whole set of parafermionic states can be generated from $\left|\sigma_{k}\right\rangle$ by the action of the modes $A_{u}$ and $A_{v}^{\dagger}$. Since the fractional part of the modes is fixed unambiguously by the charge of the field or the state on which it acts, it will be omitted in the following in order to lighten the notation. To emphasis the fact that the fractional part has been omitted, we will write $\mathcal{A}_{n}$ for $A_{n+(1+q) / N}$ and $\mathcal{B}_{m}$ for $A_{m+(1-q) / N}^{\dagger}$; more precisely, we have:

$$
\mathcal{A}_{n}\left|\phi_{[q, \bar{q}]}\right\rangle \equiv A_{n+(1+q) / N}\left|\phi_{[q, \bar{q}]}\right\rangle, \quad \mathcal{B}_{n}\left|\phi_{[q, \bar{q}]}\right\rangle \equiv A_{n+(1-q) / N}^{\dagger}\left|\phi_{[q, \bar{q}]}\right\rangle
$$

The commutation relations take the simplified forms

$$
\begin{aligned}
& \sum_{l=0}^{\infty} c_{l}\left[\mathcal{A}_{n-l-1} \mathcal{B}_{m+l+1}+\mathcal{B}_{m-l} \mathcal{A}_{n+l}\right] \phi_{[q, \bar{q}]}(0) \\
& =\left[\frac{(N+2)}{N} L_{n+m}+\frac{1}{2}\left(n+\frac{q}{N}\right)\left(n-1+\frac{q}{N}\right) \delta_{n+m, 0}\right] \phi_{[q, \bar{q}]}(0)
\end{aligned}
$$

and

$$
\sum_{l=0}^{\infty} c_{l}^{\prime}\left[\mathcal{A}_{n-l} \mathcal{A}_{m+l}-\mathcal{A}_{m-l} \mathcal{A}_{n+l}\right] \phi_{[q, \bar{q}]}(0)=0
$$

From $\left|\sigma_{k}\right\rangle$, we can generate charged sectors by the multiple action of $\mathcal{A}_{-1}$ or $\mathcal{B}_{0}$. For simplicity, we will focus on the holomorphic sector and write

$$
\sigma_{k}=\varphi_{k}^{(0)} \bar{\varphi}_{k}^{(0)}
$$

The repeated action of the $\mathcal{A}_{-1}$ operators on $\left|\varphi_{k}^{(0)}\right\rangle$ produces the states

$$
\left|\varphi_{k}^{(\ell)}\right\rangle=\mathcal{A}_{-1}^{\ell}\left|\varphi_{k}^{(0)}\right\rangle \quad 0 \leq \ell \leq N-k
$$

$\left(\mathcal{A}_{-1}^{\ell} \equiv\left(\mathcal{A}_{-1}\right)^{\ell}\right)$, with charge $2 \ell+k$ and dimension

$$
h_{\varphi_{k}^{(\ell)}}=h_{\sigma_{k}}+\frac{\ell(N-k-\ell)}{N}
$$

By acting with various powers of $\mathcal{B}_{0}$ on $\left|\varphi_{k}^{(0)}\right\rangle$, one gets

$$
\left|\varphi_{k}^{(-\ell)}\right\rangle=\mathcal{B}_{0}^{\ell}\left|\varphi_{k}^{(0)}\right\rangle \quad 0 \leq \ell \leq k
$$

of charge $-2 \ell+k$ and dimension

$$
h_{\varphi_{k}^{(-\ell)}}=h_{\sigma_{k}}+\frac{\ell(k-\ell)}{N}
$$


(The bounds on $\ell$ are explained below.) In particular, we have

$$
\psi_{k}=\varphi_{0}^{(2 k)} \quad \text { and } \quad \mu_{k}=\varphi_{k}^{(-k)} \bar{\varphi}_{k}^{(0)}
$$

The parafermionic field $\psi_{k}$ can thus be obtained from $k$ applications of the generator $\mathcal{A}_{-1}$ on the identity field. This is the announced result that the different $\psi_{k}$ can all be obtained from $\psi_{1}$. On the other hand, notice that the disorder fields $\mu_{k}$ appear themselves as descendents of the spin fields. We will see in the next section how this can be reconciliated with their primary character. Note that the states $\left|\varphi_{k}^{( \pm \ell)}\right\rangle$ are necessarily Virasoro highestweight states. It should be recalled that $\varphi_{k}^{( \pm \ell)}$ has holomorphic charge $\pm 2 \ell+k$.

\section{Field content and field identifications}

Let us first comment on the upper bounds for the value of $\ell$ in (2.23) and (2.25): these limits are fixed by the existence of singular vectors in the cases where $\ell=N-k+1$ and $\ell=k+1$ respectively. More precisely, $\left|\sigma_{k}\right\rangle$ has the following two singular vectors (the proof of this statement is reported to section 5):

$$
\mathcal{A}_{-1}^{N-k+1}\left|\sigma_{k}\right\rangle=0, \quad \mathcal{B}_{0}^{k+1}\left|\sigma_{k}\right\rangle=0
$$

to which correspond the null fields

$$
\varphi_{k}^{(N-k+1)}=0, \quad \varphi_{k}^{(-k-1)}=0
$$

Therefore the two states at the extremity of the string $\left|\varphi_{k}^{(\ell)}\right\rangle$ satisfy

$$
\mathcal{A}_{-1}\left|\varphi_{k}^{(N-k)}\right\rangle=0, \quad \mathcal{B}_{0}\left|\varphi_{k}^{(-k)}\right\rangle=0
$$

Rewritting this with the fractional part of the modes reinserted, this is nothing but

$$
\mathcal{A}_{1+(1-k) / N}\left|\varphi_{k}^{(N-k)}\right\rangle=0, \quad \mathcal{B}_{(1+k) / N}\left|\varphi_{k}^{(-k)}\right\rangle=0
$$

Now, the two fields $\varphi_{k}^{(N-k)}$ and $\varphi_{k}^{(-k)}$ have the same dimension and their charge differ by $2 N$. It has already been indicated that the charge is defined modulo $2 N$. The parafermionic theory under consideration is essentially an affine $\widehat{s u}(2)$ theory, up to the description of the charge (this statement is made precise below, in terms of a coset) and for such theories, the 
equality of the conformal dimension ensures the field identification. Therefore, the fields $\varphi_{k}^{(N-k)}$ and $\varphi_{k}^{(-k)}$ are identical:

$$
\varphi_{k}^{(N-k)}=\varphi_{k}^{(-k)}
$$

Since $\varphi_{k}^{(-k)}=\mu_{k}$, these are two representations of the disorder field. The singular-vector expressions (3.4) are thus simply

$$
\mathcal{A}_{1+(1-k) / N}\left|\mu_{k}\right\rangle=\mathcal{B}_{(1+k) / N}\left|\mu_{k}\right\rangle=0
$$

The other positive modes annihilate $\left|\mu_{k}\right\rangle$ (this is made explicit in section 5). As a result, the singular-vector conditions are nothing but the highest-weight conditions (2.18) on the disorder states.

From an heuristic point of view, one can see the existence of these singular vectors as ensuring the unitarity of the theory, that is, the absence of fields with negative conformal dimension. Indeed, observe that by writting explicitly the fractional modes in $\mathcal{A}_{-1}^{N-k+1}\left|\sigma_{k}\right\rangle$, we get

$$
A_{1+(1-k) / N}\left[A_{1-(1+k) / N} \cdots A_{-1+(1+k) / N}\right]\left|\sigma_{k}\right\rangle
$$

and the sum of the fractional modes of the $N-k$ rightmost factors add up to zero (which is also clear from the fact that $\mathcal{A}_{-1}^{N-k}\left|\sigma_{k}\right\rangle=\left|\mu_{k}\right\rangle$ and that $h_{\sigma_{k}}=h_{\mu_{k}}$ ). The dimension of the extra mode is such that when added up to that of $\sigma_{k}$, the sum is negative. In other words, in the string $\mathcal{A}_{-1}^{\ell}\left|\sigma_{k}\right\rangle$, as $\ell$ increases, the dimension starts by increasing but at some point this process is reversed and it then decreases continuously; however, the very first state that acquires a negative dimension is singular. 目 The same remark applies to the $\mathcal{B}_{0}^{\ell}\left|\sigma_{k}\right\rangle$ string.

In the holomorphic sector, $\mu_{k}$ is identical to $\sigma_{N-k}$ : they have the same dimension and satisfiy the same highest-weight conditions. More precisely, $\varphi_{N-k}^{(0)}=\varphi_{k}^{(-k)}$. Therefore, by including all the spin primary states and their descendents, we count twice too many states.

To avoid this double counting, we can thus restrict the space of fields to those obtained from $\left|\varphi_{k}^{(0)}\right\rangle$ and their descendents, with $k=0, \cdots,[N / 2]$, (the square bracket indicates the

3 If the $\mathcal{A}$-string contains indices less than -1 , more $\mathcal{A}_{-1}$ operators are needed in order to reach a negative dimension and these additional operators ensure that the resulting string can be described as a descendent of the singular vector. 
integer part) when $N$ is odd and include half of the descendents of $\left|\varphi_{N / 2}^{(0)}\right\rangle$ when $N$ is even. More simply, we could avoid considering both the $\mathcal{A}$-type and the $\mathcal{B}$-type descendents and keep only one of these two sets. In that case, we should not keep the 'holomorphic disorder fields' as 'holomorphic spin-field' descendents since they already appear in disguised form as $\varphi_{N-k}^{(0)}$. For instance, if we keep only the $\mathcal{A}$ descendents, then we need to keep all the $\mathcal{A}$ descendents of $\left|\varphi_{k}^{(0)}\right\rangle$ up to the next to last one (since the last one is the holomorphic restriction of the disorder state, $\left.\left|\varphi_{k}^{(-k)}\right\rangle\right)$. The resulting set of independent states $\left|\varphi_{k}^{(\ell)}\right\rangle$ is thus:

$$
\left\{\left|\varphi_{k}^{(\ell)}\right\rangle=\mathcal{A}_{-1}^{\ell}\left|\varphi_{k}^{(0)}\right\rangle \mid k=0, \cdots N-1, \quad 0 \leq \ell \leq N-k-1\right\}
$$

The number of such states is simply

$$
\sum_{k=0}^{N-1} \sum_{\ell=0}^{N-1-k} 1=\frac{N(N+1)}{2}
$$

This counting of states can be checked by considering coset realizations of this parafermionic model. The simplest one is $\widehat{s u}(2)_{N} / \widehat{u}(1)$. The number of independent primary coset fields is also $N(N+1) / 2$ (cf. [10] sect.18.5). Another one is furnished by the diagonal coset $\widehat{s u}(N)_{1} \oplus \widehat{s u}(N)_{1} / \widehat{s u}(N)_{2}$. The number of distinct holomorphic WZW primary fileds for the $\widehat{s u}(N)_{k}$ model is $(k+N-1) ! / k !(N-1)$ !. The branching condition in the coset is simply taken into account by ignoring one $\widehat{s u}(N)_{1}$ factor (there is a unique field associated to this WZW term that is compatible with the branching condition of the three coset component-fields). The total number of coset fields must be reduced by a factor $N$ to take care of the field identifications resulting from outer automorphisms (and there are no fixed points here). The number of distinct coset fields is thus:

$$
\frac{N !}{(N-1) !} \times \frac{(N+1) !}{2 !(N-1) !} \times \frac{1}{N}=\frac{N(N+1)}{2}
$$

Let us list the independent holomorphic fields $\varphi_{k}^{(\ell)}$ for the first few values of $N$. For $N=2$, which is the Ising model, we have the following correspondence with Virasoro primary fields

$$
\begin{array}{ll}
\varphi_{0}^{(0)}=I=\phi_{(1,1)} & \varphi_{0}^{(1)}=\psi=\phi_{(2,1)} \\
(h=0) & (h=1 / 2) \\
\varphi_{1}^{(0)}=\sigma=\phi_{(1,2)} & \\
(h=1 / 16) &
\end{array}
$$


(where here and below the spin-field notation refers to its holomorphic restriction). The $N=3$ parafermionic theory corresponds to the three-state Potts model (whose Virasoro partition function has a non-diagonal form):

$$
\begin{array}{lll}
\varphi_{0}^{(0)}=I=\phi_{(1,1)}+\phi_{(4,1)} & \varphi_{0}^{(1)}=\psi_{1}=\phi_{(1,3)} & \varphi_{0}^{(2)}=\psi_{2}=\phi_{(1,3)}^{*} \\
(h=0) & (h=2 / 3) & (h=2 / 3) \\
\varphi_{1}^{(0)}=\sigma_{1}=\phi_{(2,3)} & \varphi_{1}^{(1)}=\epsilon_{1}=\phi_{(2,1)}+\phi_{(3,1)} & \\
(h=1 / 15) & (h=2 / 5) & \\
\varphi_{2}^{(0)}=\sigma_{2}=\phi_{(2,3)}^{*} & \\
(h=1 / 15) &
\end{array}
$$

The $N=4$ model is an orbifold $c=1$ theory on a rational circle of radius $R=\sqrt{6}$, with field content:

$$
\begin{aligned}
& \varphi^{(0)}=I \\
& \varphi_{0}^{(1)}=\Phi_{3}^{(1)} \\
& \varphi_{0}^{(2)}=\Theta \\
& \varphi_{0}^{(3)}=\Phi_{3}^{(2)} \\
& (h=0) \\
& (h=3 / 4) \\
& (h=1) \\
& (h=3 / 4) \\
& \varphi_{1}^{(0)}=\sigma^{(1)} \\
& \varphi_{1}^{(1)}=\tau^{(1)} \\
& \varphi_{1}^{(2)}=\tau^{(2)} \\
& (h=1 / 16) \\
& (h=9 / 16) \\
& (h=9 / 16) \\
& \varphi_{2}^{(0)}=\Phi_{1} \\
& \varphi_{2}^{(1)}=\Phi_{2} \\
& (h=1 / 12) \\
& (h=1 / 3) \\
& \varphi_{3}^{(0)}=\sigma^{(2)} \\
& (h=1 / 16)
\end{aligned}
$$

(compare with [11] and [10] sect. 17.B.6). Here $\sigma^{(i)}$ and $\tau^{(i)}(i=1,2)$ are the twist fields of the orbifold theory and $\Theta$ is a dimension-one field. The rest of the spectrum depends upon the value of the radius; with $R=\sqrt{2 p^{\prime}}$, there are the fields $\Phi_{\lambda}$ with $\lambda=1,2, \cdots, p^{\prime}-1$ of dimension $\lambda^{2} / 4 p^{\prime}$ and the doubly degenerate field $\Phi_{p^{\prime}}^{(i)}$ of dimension $p^{\prime} / 4$.

\section{A basis of states}

We now determine a convenient basis of states for the description of a generic highestweight module in the parafermionic algebra, level by level. The highest-weight state $\left|\phi_{q}\right\rangle$ 
is defined by the condition

$$
\mathcal{A}_{-n-1}\left|\phi_{q}\right\rangle=\mathcal{B}_{-n}\left|\phi_{q}\right\rangle=L_{-n-1}\left|\phi_{q}\right\rangle=0 \quad \text { for } \quad n \geq 0
$$

As before, we ignore the fractional part of the modes. The results are presented in the form of lemma, illustrated with simple examples. The proof of the first three lemma is reported in Appendix A. For the other ones, the proof is obvious.

Lemma 1 - On a generic highest-weight state $\left|\phi_{q}\right\rangle$, any state at level $s$ built out of $j$ operators $\mathcal{A}$ can be expressed as a linear combination of terms of the form

$$
\mathcal{A}_{-n_{1}} \mathcal{A}_{-n_{2}} \ldots \mathcal{A}_{-n_{j}}\left|\phi_{q}\right\rangle
$$

where $\sum_{i} n_{i}=s \geq j$ and $n_{i} \geq 1$ and $n_{k} \geq n_{l}$ if $k<l$.

Lemma 2 - On a generic highest-weight state $\left|\phi_{q}\right\rangle$, any state at level $s$ built out of $j$ operators $\mathcal{B}$ can be expressed as a linear combination of terms of the form

$$
\mathcal{B}_{-m_{1}} \mathcal{B}_{-m_{2}} \ldots \mathcal{B}_{-m_{j}}\left|\phi_{q}\right\rangle
$$

where $\sum_{i} m_{i}=s$ and $m_{i} \geq 0$ and $m_{k} \geq m_{l}$ if $k<l$.

Consider now mixed strings, that is, sequences containing both types of operators. The rearrangement of such strings will generate $L_{m}$ terms. These are easily taken care of by noticing the relation (that follows from $(2.10)$ with $n=0)$ :

$$
L_{m}\left|\phi_{q}\right\rangle=\frac{N}{N+2} \sum_{l=0}^{\infty} c_{l}^{\prime} \mathcal{A}_{-l-1} \mathcal{B}_{m+l+1}\left|\phi_{q}\right\rangle
$$

Hence, the Virasoro modes can be replaced by appropriate sums of $\mathcal{A B}$-type terms and can thus be ignored in the counting of independent states.

Lemma 3 - On a generic highest-weight state $\left|\phi_{q}\right\rangle$, a state at level $s$ containing $j$ operators $\mathcal{A}$ and $k$ operators $\mathcal{B}$ in any ordering, can be written as a linear combination of terms of the type

$$
\mathcal{A}_{-n_{1}} \mathcal{A}_{-n_{2}} \ldots \mathcal{A}_{-n_{j^{\prime}}} \mathcal{B}_{-m_{1}} \mathcal{B}_{-m_{2}} \ldots \mathcal{B}_{-m_{k^{\prime}}}\left|\phi_{q}\right\rangle
$$

with

$$
j-k=j^{\prime}-k^{\prime}, \quad \sum_{i=1}^{j^{\prime}} n_{i}+\sum_{i=1}^{k^{\prime}} m_{i}=s
$$


and

$$
n_{i} \geq 1 \quad n_{p} \geq n_{l} \quad \text { if } \quad p<l \quad \text { and } \quad m_{i} \geq 0 \quad m_{p} \geq m_{l} \quad \text { if } \quad p<l
$$

The main point of this lemma is that we can order separately the $\mathcal{A}$ strings and the $\mathcal{B}$ strings but there is no mixed ordering.

We illustrate these results with simple examples. Consider first $\mathcal{A}_{2} \mathcal{A}_{-4}\left|\phi_{q}\right\rangle$. $\mathcal{A}_{2} \mathcal{A}_{-4}$ is the first term of the infinite sum $\sum_{l \geq 0} c_{l} \mathcal{A}_{n-l} \mathcal{A}_{m+l}$ with $n=2$ and $m=-4$, since $c_{0}=1$. All but a finite number of terms in this sum vanish when it is acted on a highest-weight state. In fact, we have:

$$
\mathcal{A}_{2} \mathcal{A}_{-4}\left|\phi_{q}\right\rangle=\left(\sum_{l=0}^{\infty} c_{l} \mathcal{A}_{2-l} \mathcal{A}_{-4+l}-c_{1} \mathcal{A}_{1} \mathcal{A}_{-3}-c_{2} \mathcal{A}_{0} \mathcal{A}_{-2}-c_{3} \mathcal{A}_{-1} \mathcal{A}_{-1}\right)\left|\phi_{q}\right\rangle
$$

Using the commutation relation (2.13), we can rewrite the infinite sum differently, as:

$$
\begin{aligned}
\mathcal{A}_{2} \mathcal{A}_{-4}\left|\phi_{q}\right\rangle & =\left(\sum_{l=0}^{\infty} c_{l} \mathcal{A}_{-4-l} \mathcal{A}_{2+l}-c_{1} \mathcal{A}_{1} \mathcal{A}_{-3}-c_{2} \mathcal{A}_{0} \mathcal{A}_{-2}-c_{3} \mathcal{A}_{-1} \mathcal{A}_{-1}\right)\left|\phi_{q}\right\rangle \\
& =\left(-c_{1} \mathcal{A}_{1} \mathcal{A}_{-3}-c_{2} \mathcal{A}_{0} \mathcal{A}_{-2}-c_{3} \mathcal{A}_{-1} \mathcal{A}_{-1}\right)\left|\phi_{q}\right\rangle
\end{aligned}
$$

Similarly, $\mathcal{A}_{1} \mathcal{A}_{-3}\left|\phi_{q}\right\rangle$ can be expressed as a linear combination of $\mathcal{A}_{0} \mathcal{A}_{-2}\left|\phi_{q}\right\rangle$ and $\mathcal{A}_{-1} \mathcal{A}_{-1}\left|\phi_{q}\right\rangle$ and finally, $\mathcal{A}_{0} \mathcal{A}_{-2}\left|\phi_{q}\right\rangle$ is found to be proportional to $\mathcal{A}_{-1} \mathcal{A}_{-1}\left|\phi_{q}\right\rangle$. Hence, $\mathcal{A}_{2} \mathcal{A}_{-4}\left|\phi_{q}\right\rangle$ is simply proportional to $\mathcal{A}_{-1} \mathcal{A}_{-1}\left|\phi_{q}\right\rangle$, as expected, since the latter is the only independent state at level 2 with relative charge 4 .

Consider next $\mathcal{B}_{-3} \mathcal{A}_{-1}\left|\phi_{q}\right\rangle$; since $c_{0}^{\prime}=1$, we can write

$$
\begin{aligned}
\mathcal{B}_{-3} \mathcal{A}_{-1}\left|\phi_{q}\right\rangle & =\sum_{l=0}^{\infty} c_{l}^{\prime} \mathcal{B}_{-3-l} \mathcal{A}_{-1+l}\left|\phi_{q}\right\rangle \\
& =\left[-\sum_{l=0}^{\infty} c_{l}^{\prime} \mathcal{A}_{-2-l} \mathcal{B}_{-2+l}+\frac{N+2}{N} L_{-4}\right]\left|\phi_{q}\right\rangle \\
& =\left[-\mathcal{A}_{-2} \mathcal{B}_{-2}-c_{1}^{\prime} \mathcal{A}_{-3} \mathcal{B}_{-1}-c_{2}^{\prime} \mathcal{A}_{-4} \mathcal{B}_{0}+\frac{N+2}{N} L_{-4}\right]\left|\phi_{q}\right\rangle
\end{aligned}
$$

And using the expression (4.4):

$$
\frac{N+2}{N} L_{-4}\left|\phi_{q}\right\rangle=\left[\mathcal{A}_{-1} \mathcal{B}_{-3}+c_{1}^{\prime} \mathcal{A}_{-2} \mathcal{B}_{-2}+c_{2}^{\prime} \mathcal{A}_{-3} \mathcal{B}_{-1}+c_{3}^{\prime} \mathcal{A}_{-4} \mathcal{B}_{0}\right]\left|\phi_{q}\right\rangle
$$


so that

$$
\mathcal{B}_{-3} \mathcal{A}_{-1}\left|\phi_{q}\right\rangle=\left[\mathcal{A}_{-1} \mathcal{B}_{-3}+\left(c_{1}^{\prime}-1\right) \mathcal{A}_{-2} \mathcal{B}_{-2}+\left(c_{2}^{\prime}-c_{1}^{\prime}\right) \mathcal{A}_{-3} \mathcal{B}_{-1}+\left(c_{3}^{\prime}-c_{2}^{\prime}\right) \mathcal{A}_{-4} \mathcal{B}_{0}\right]\left|\phi_{q}\right\rangle
$$

and this is indeed a linear combination of all the independent states identified in lemma 3.

Consider finally $\mathcal{A}_{-1} \mathcal{A}_{-2} \mathcal{B}_{0}\left|\phi_{q}\right\rangle$. Observe that $\mathcal{A}_{p} \mathcal{B}_{0}\left|\phi_{q}\right\rangle=0$ if $p>0$. Indeed, it can be written as the $l=0$ term of the sum $\sum_{l \geq 0} c_{l}^{\prime} \mathcal{A}_{n-1-l} \mathcal{B}_{m+1+l}\left|\phi_{q}\right\rangle$ with $n=p+1$ and $m=-1$; this sum can be inverted by means of (2.10) to yield $-\sum_{l \geq 0} c_{l}^{\prime} \mathcal{B}_{-1-l} \mathcal{A}_{p+1+l}\left|\phi_{q}\right\rangle$, and all these terms vanish, plus a term proportional to $L_{p}\left|\phi_{q}\right\rangle$, which is also zero. With this information, we can write

$$
\begin{aligned}
\mathcal{A}_{-1} \mathcal{A}_{-2} \mathcal{B}_{0}\left|\phi_{q}\right\rangle & =\left[\sum_{l=0}^{\infty} c_{l} \mathcal{A}_{-1-l} \mathcal{A}_{-2+l}-c_{1} \mathcal{A}_{-2} \mathcal{A}_{-1}-c_{2} \mathcal{A}_{-3} \mathcal{A}_{0}\right] \mathcal{B}_{0}\left|\phi_{q}\right\rangle \\
& =\left[\sum_{l=0}^{\infty} c_{l} \mathcal{A}_{-2-l} \mathcal{A}_{-1+l}-c_{1} \mathcal{A}_{-2} \mathcal{A}_{-1}-c_{2} \mathcal{A}_{-3} \mathcal{A}_{0}\right] \mathcal{B}_{0}\left|\phi_{q}\right\rangle \\
& =\left[\left(1-c_{1}\right) \mathcal{A}_{-2} \mathcal{A}_{-1}-\left(c_{1}-c_{2}\right) \mathcal{A}_{-3} \mathcal{A}_{0}\right] \mathcal{B}_{0}\left|\phi_{q}\right\rangle
\end{aligned}
$$

Finally, we have

$$
\mathcal{A}_{0} \mathcal{B}_{0}\left|\phi_{q}\right\rangle=\frac{N+2}{N} L_{0}\left|\phi_{q}\right\rangle=\frac{N+2}{N} h_{\phi_{q}}\left|\phi_{q}\right\rangle
$$

so that when acting on a highest-weight state, $\mathcal{A}_{-1} \mathcal{A}_{-2} \mathcal{B}_{0}$ can be expressed as a linear combination of $\mathcal{A}_{-2} \mathcal{A}_{-1} \mathcal{B}_{0}$ and $\mathcal{A}_{-3}$.

We stress that the sum of the fractional parts of the modes in a mixed string does not depend upon the relative position of the $\mathcal{A}$ and $\mathcal{B}$ operators in the string; it depends only on their relative number. More precisely, for the string (4.6), this sum is equal to

$$
\frac{1}{N}(j-k)[q+(j-k)]
$$

Consider now the counting of states:

Lemma 4 - The number of independent states of type $\mathcal{A}$ and lenght $j$ of the form $\mathcal{A}_{-n_{1}} \ldots \mathcal{A}_{-n_{j}}$ with $n_{i} \geq 1, n_{k} \geq n_{l}$ if $k<l$ and $\sum n_{i}=s$, is given by the number of partitions of the positive integer $s$ of lenght $j$, denoted as $p^{[j]}(s)$.

In other words, $p^{[j]}(s)$ is the number of partitions of $s$ with exactly $j$ parts. For instance $p^{[2]}(8)=4$ since 8 can be decomposed in a partition of two terms in four ways: $7+1=6+2=5+3=4+4$. 
Lemma 5 - The number of independent of states of type $\mathcal{B}$ and lenght $j$ of the form $\mathcal{B}_{-m_{1}} \ldots \mathcal{B}_{-m_{j}}$ with $m_{i} \geq 0, m_{k} \geq m_{l}$ if $k<l$ and $\sum m_{i}=s$, is given by the number of partitions of the positive integer $s$ with at most $j$ parts, denoted as $p^{(j)}(s)$.

There is an obvious relation between partitions having exactly $j$ parts and those of having at most $j$ parts:

$$
p^{(j)}(s)=\sum_{i=1}^{j} p^{[i]}(s)
$$

For instance $p^{(2)}(8)=5$ since 8 has precisely one partition into one part, 8 itself, that contributes in addition to the 4 two-part partitions given above.

In fact, there is an even simpler relation between the type of partitions that enter in the counting of the $\mathcal{A}$ states and the ones used for counting the $\mathcal{B}$ states:

$$
p^{[j]}(s)=p^{(j)}(s-j)
$$

For instance, $p^{(3)}(5)=5$ since 5 can be decomposed in 5 different ways in sums of at most three integers: $5=4+1=3+2=3+1+1=2+2+1$, and we have $p^{[3]}(8)=5$ since there are 5 decompositions of 8 into 3 integers (obtained from the above ones by adding a 1 to each partition - after incorporating an appropriate number of zeros -; for example, $4+1$ is first rewritten as $4+1+0$ and then transformed to $5+2+1$.

Lemma 6 - The number of states

$$
\mathcal{A}_{-n_{1}} \mathcal{A}_{-n_{2}} \ldots \mathcal{A}_{-n_{j}} \mathcal{B}_{-m_{1}} \mathcal{B}_{-m_{2}} \ldots \mathcal{B}_{-m_{k}}\left|\phi_{q}\right\rangle
$$

with

$$
j-k=r \quad \sum_{i=1}^{j} n_{i}=s_{1}, \quad \sum_{i=1}^{k} m_{i}=s_{2}, \quad s_{1}+s_{2}=s,
$$

with $r, s$ fixed (and $r$ supposed to be positive), is given by

$$
\sum_{j=0}^{s} \sum_{s_{1}=j}^{s} p^{(j)}\left(s_{1}-j\right) p^{(j-r)}\left(s-s_{1}\right)
$$

If $r$ is negative, the product $p^{(j)}\left(s_{1}-j\right) p^{(j-r)}\left(s-s_{1}\right)$ is replaced by $p^{(j-|r|)}\left(s_{1}-\right.$ j) $p^{(j)}\left(s-s_{1}\right)$. However, it is not necessary to treat both cases separately since there is a charge reversal symmetry: the counting of states is independent of the sign of the relative charge. 
For the restricted partitions, we use the conventions

$$
\begin{array}{ll}
p^{(0)}(n)=\delta_{n, 0} & \\
p^{(j)}(0)=1 & \text { for } \quad j \geq 0 \\
p^{(j)}(n)=0 & \text { for } \quad j<0, \forall n
\end{array}
$$

Useful expressions are:

$$
\begin{aligned}
& p^{(1)}(n)=1 \\
& p^{(2)}(n)=\left[\frac{n+2}{2}\right] \\
& p^{(n-1)}(n)=p(n)-1 \\
& p^{(j)}(n)=p(n) \quad \text { if } \quad j \geq n
\end{aligned}
$$

(where, as before, the square bracket indicates the integer part). To illustrate the above formula, we list the states for $1 \leq s \leq 3$ and $r=0$ :

$$
\begin{array}{cl}
s=1: \mathcal{A}_{-1} \mathcal{B}_{0} & p^{(1)}(0) p^{(1)}(0)=1 \\
s=2: \mathcal{A}_{-1} \mathcal{B}_{-1} & p^{(0)}(1) p^{(1)}(1)=1 \\
\mathcal{A}_{-2} \mathcal{B}_{0} & p^{(1)}(1) p^{(1)}(0)=1 \\
\mathcal{A}_{-1} \mathcal{A}_{-1} \mathcal{B}_{0} \mathcal{B}_{0} & p^{(2)}(0) p^{(2)}(0)=1 \\
s=3: \mathcal{A}_{-1} \mathcal{B}_{-2} & p^{(1)}(0) p^{(1)}(2)=1 \\
\mathcal{A}_{-2} \mathcal{B}_{-1} & p^{(1)}(1) p^{(1)}(1)=1 \\
\mathcal{A}_{-3} \mathcal{B}_{0} & p^{(1)}(2) p^{(1)}(0)=1 \\
\mathcal{A}_{-1} \mathcal{A}_{-1} \mathcal{B}_{-1} \mathcal{B}_{0} & p^{(2)}(0) p^{(2)}(1)=1 \\
\mathcal{A}_{-2} \mathcal{A}_{-1} \mathcal{B}_{0} \mathcal{B}_{0} & p^{(2)}(1) p^{(2)}(0)=1 \\
\mathcal{A}_{-1} \mathcal{A}_{-1} \mathcal{A}_{-1} \mathcal{B}_{0} \mathcal{B}_{0} \mathcal{B}_{0} & p^{(3)}(0) p^{(3)}(0)=1
\end{array}
$$

Clearly, the $j=0$ term in the sum contributes only when $s=0$. At a generic level $s$, the contribution of the $j=1$ term is simply

$$
\sum_{s_{1}=1}^{s} p^{(1)}\left(s_{1}-1\right) p^{(1)}\left(s-s_{1}\right)=s
$$

since $p^{(1)}(n)=1$ for all $n \geq 1$. On the other hand, the contribution of the $j=s$ term, which forces $s_{1}=j$, is simply $p^{(s)}(0) p^{(s)}(0)=1$. The sum (4.20) can thus be rewritten somewhat more simply as (still pursuing the illustration of the $r=0$ case):

$$
s+1+\sum_{j=2}^{s-1} \sum_{s_{1}=j}^{s} p^{(j)}\left(s_{1}-j\right) p^{(j)}\left(s-s_{1}\right)
$$


For $s=4$, it is simple to check that there are 12 terms (grouped by their value of $j$ ordered as in the above expression):

$$
\begin{aligned}
5 & +\left[p^{(2)}(0) p^{(2)}(2)+p^{(2)}(1) p^{(2)}(1)+p^{(2)}(2) p^{(2)}(0)\right] \\
& +\left[p^{(3)}(0) p^{(3)}(1)+p^{(3)}(1) p^{(3)}(0)\right]=5+[2+1+2]+[1+1]=12
\end{aligned}
$$

For instance, the two states associated to $p^{(2)}(2) p^{(2)}(0)=2 \cdot 1$ are $\mathcal{A}_{-3} \mathcal{A}_{-1} \mathcal{B}_{0} \mathcal{B}_{0}$ and $\mathcal{A}_{-2} \mathcal{A}_{-2} \mathcal{B}_{0} \mathcal{B}_{0}$

If the relative charge $2 r$ is non-zero, the first level $s$ at which the sum is nonvanishing is $s=r$ since $p^{(j-r)}(n)=0$ if $j<r$, irrespectively of the value of $n$. This first non-zero term is associated to a unique state since $p^{(r)}(0) p^{(0)}(0)=1$.

\section{Parafermionic singular vectors}

We will now prove that every parafermionic highest-weight state $\left|\sigma_{k}\right\rangle$ has two (primary) holomorphic singular vectors, given by

$$
\left|\chi_{N-k+1}\right\rangle=\mathcal{A}_{-1}^{N-k+1}\left|\sigma_{k}\right\rangle, \quad\left|\chi_{k+1}^{\prime}\right\rangle=\mathcal{B}_{0}^{k+1}\left|\sigma_{k}\right\rangle
$$

We thus want to prove that

$$
\mathcal{A}_{p}|\xi\rangle=\mathcal{B}_{p+1}|\xi\rangle=L_{p+1}|\xi\rangle=0 \quad \text { for } \quad p \geq 0
$$

for $|\xi\rangle=\left|\chi_{N-k+1}\right\rangle$ and $\left|\chi_{k+1}^{\prime}\right\rangle$.

Let us first consider the $|\chi\rangle$-type singular vector and start with the action of the $\mathcal{A}_{p}$ modes for $p \geq 0$. Consider first their action on $\mathcal{A}_{-1}\left|\sigma_{k}\right\rangle$ :

$$
\mathcal{A}_{p} \mathcal{A}_{-1}\left|\sigma_{k}\right\rangle=\sum_{l=0}^{\infty} c_{l}^{\prime} \mathcal{A}_{p-l} \mathcal{A}_{-1+l}\left|\sigma_{k}\right\rangle=\sum_{l=0}^{\infty} c_{l}^{\prime} \mathcal{A}_{-1-l} \mathcal{A}_{p+l}\left|\sigma_{k}\right\rangle=0
$$

In the first step we used the fact that only the $l=0$ term does contribute to the sum (and $c_{0}^{\prime}=1$ ); the second equality follows from (2.13) and all the terms of this second sum

4 For simplicity, in this section we stick to the $\sigma$ notation, but we focus on its holomorphic singular vectors. 
vanish on a highest-weight state. Now suppose that $\mathcal{A}_{p} \mathcal{A}_{-1}^{n}\left|\sigma_{k}\right\rangle=0$ for a general value of $n$, then

$$
\mathcal{A}_{p} \mathcal{A}_{-1}^{n+1}\left|\sigma_{k}\right\rangle=\sum_{l=0}^{\infty} c_{l}^{\prime} \mathcal{A}_{p-l} \mathcal{A}_{-1+l} \mathcal{A}_{-1}^{n}\left|\sigma_{k}\right\rangle=\sum_{l=0}^{\infty} c_{l}^{\prime} \mathcal{A}_{-1-l} \mathcal{A}_{p+l} \mathcal{A}_{-1}^{n}\left|\sigma_{k}\right\rangle=0
$$

In view of the recursive hypothesis, only the $l=0$ term does contribute in the first sum, while in the second sum all terms vanish. We have thus shown that $\mathcal{A}_{p} \mathcal{A}_{-1}^{n+1}\left|\sigma_{k}\right\rangle=0$ for all values of $n$.

Consider now the action of $L_{p}$ for $p>0$ on $\mathcal{A}_{-1}^{n+1}\left|\sigma_{k}\right\rangle$. For $n=0$, we have

$$
L_{p} \mathcal{A}_{-1}\left|\sigma_{k}\right\rangle=\mathcal{A}_{-1} L_{p}\left|\sigma_{k}\right\rangle+\frac{(p+1+k-N)}{N} A_{p-1}\left|\sigma_{k}\right\rangle
$$

and both terms vanish due to the highest-weight conditions. Suppose that $L_{p} \mathcal{A}_{-1}^{n}\left|\sigma_{k}\right\rangle=0$ for a general value of $n$, then

$$
L_{p} \mathcal{A}_{-1}^{n+1}\left|\sigma_{k}\right\rangle=\mathcal{A}_{-1} L_{p} \mathcal{A}_{-1}^{n}\left|\sigma_{k}\right\rangle+\frac{(p+1+k+2 n-N)}{N} A_{p-1} \mathcal{A}_{-1}^{n}\left|\sigma_{k}\right\rangle=0
$$

Indeed, the first term vanishes by the recursion hypothesis and the second vanishes due to $(5.4)$.

Finally, we consider the action of the $\mathcal{B}_{p}$ operators. We start with $p>1$ and leave for a separate treatment the special case $p=1$. We have (with $c_{0}=1$ )

$$
\mathcal{B}_{p} \mathcal{A}_{-1}\left|\sigma_{k}\right\rangle=\sum_{l=0}^{\infty} c_{l} \mathcal{B}_{p-l} \mathcal{A}_{-1+l}\left|\sigma_{k}\right\rangle=-\sum_{l=0}^{\infty} c_{l} \mathcal{A}_{-2-l} \mathcal{B}_{p+l+1}\left|\sigma_{k}\right\rangle+\frac{N+2}{N} L_{p-1}\left|\sigma_{k}\right\rangle=0
$$

Indeed, all the terms in the second sum vanish by the highest-weight condition and similarly $L_{p-1}\left|\sigma_{k}\right\rangle=0$. Again we suppose that $\mathcal{B}_{p} \mathcal{A}_{-1}^{n}\left|\sigma_{k}\right\rangle=0$ for a general value of $n$, and prove the result for $n+1$, by standard steps:

$$
\begin{aligned}
\mathcal{B}_{p} \mathcal{A}_{-1}^{n+1}\left|\sigma_{k}\right\rangle & =\sum_{l=0}^{\infty} c_{l} \mathcal{B}_{p-l} \mathcal{A}_{-1+l} \mathcal{A}_{-1}^{n}\left|\sigma_{k}\right\rangle \\
& =-\sum_{l=0}^{\infty} c_{l} \mathcal{A}_{-2-l} \mathcal{B}_{p+l+1} \mathcal{A}_{-1}^{n}\left|\sigma_{k}\right\rangle+\frac{N+2}{N} L_{p-1} \mathcal{A}_{-1}^{n}\left|\sigma_{k}\right\rangle=0
\end{aligned}
$$

The vanishing of the first term in the last line follows from the recursive hypothesis and the second one vanishes due to (5.6). 
At this point, we have not found any constraint on the value of $n$, i.e., $\mathcal{A}_{-1}^{n+1}\left|\sigma_{k}\right\rangle$ satisfies identically all the highest-weight conditions considered so far. The constraint on the value of $n$ follows from the consideration of the action of the $\mathcal{B}_{1}$ mode. Consider then

$$
\begin{aligned}
\mathcal{B}_{1} \mathcal{A}_{-1}\left|\sigma_{k}\right\rangle & =\sum_{l=0}^{\infty} c_{l} \mathcal{B}_{1-l} \mathcal{A}_{-1+l}\left|\sigma_{k}\right\rangle \\
& =-\sum_{l=0}^{\infty} c_{l} \mathcal{A}_{-2-l} \mathcal{B}_{2+l}\left|\sigma_{k}\right\rangle+\left[\frac{N+2}{N} L_{0}+\frac{1}{2}\left(-1+\frac{k}{N}\right)\left(-2+\frac{k}{N}\right)\right]\left|\sigma_{k}\right\rangle \\
& =\left[\frac{N+2}{N} h_{\sigma_{k}}+\frac{1}{2}\left(-1+\frac{k}{N}\right)\left(-2+\frac{k}{N}\right)\right]\left|\sigma_{k}\right\rangle \\
& =\frac{N-k}{N}\left|\sigma_{k}\right\rangle
\end{aligned}
$$

This vanishes only if $N=k$. More generally, consider

$$
\begin{aligned}
\mathcal{B}_{1} \mathcal{A}_{-1}^{n+1}\left|\sigma_{k}\right\rangle= & \sum_{l=0}^{\infty} c_{l} \mathcal{B}_{1-l} \mathcal{A}_{-1+l} \mathcal{A}_{-1}^{n}\left|\sigma_{k}\right\rangle \\
= & -\sum_{l=0}^{\infty} c_{l} \mathcal{A}_{-2-l} \mathcal{B}_{l+2} \mathcal{A}_{-1}^{n}\left|\sigma_{k}\right\rangle \\
& +\left[\frac{N+2}{N} L_{0}+\frac{1}{2}\left(-1+\frac{k^{\prime}}{N}\right)\left(-2+\frac{k^{\prime}}{N}\right)\right] \mathcal{A}_{-1}^{n}\left|\sigma_{k}\right\rangle
\end{aligned}
$$

The different terms in the last sum vanish when acting on $\left|\sigma_{k}\right\rangle$ due to (5.8). The value of $k^{\prime}$ that appears in the last part refers to the charge of the state at its right: hence, $k^{\prime}=k+2 n$. The eigenvalue of $L_{0}$ is easily found to be

$$
L_{0} \mathcal{A}_{-1}^{n}\left|\sigma_{k}\right\rangle=\left[n-\frac{1}{N} n(n+k)+h_{\sigma_{k}}\right] \mathcal{A}_{-1}^{n}\left|\sigma_{k}\right\rangle
$$

(for this computation, the fractional part of the modes has to be reinserted.) Substituting the value of $h_{\sigma_{k}}$, we are thus left with

$$
\mathcal{B}_{1} \mathcal{A}_{-1}^{n+1}\left|\sigma_{k}\right\rangle=\frac{(n+1)(N-k-n)}{N} \mathcal{A}_{-1}^{n+1}\left|\sigma_{k}\right\rangle
$$

which is zero only if $n=N-k$.

The analysis of the other singular vector is similar and it will not be detailed. Suffice to mention that

$$
\mathcal{B}_{p} \mathcal{B}_{0}^{n+1}\left|\sigma_{k}\right\rangle=L_{p} \mathcal{B}_{0}^{n+1}\left|\sigma_{k}\right\rangle=\mathcal{A}_{p} \mathcal{B}_{0}^{n+1}\left|\sigma_{k}\right\rangle \quad p \geq 1
$$


for all values of $n$. The constraint on $n$ follows from considering the action of $\mathcal{A}_{0}$ :

$$
\mathcal{A}_{0} \mathcal{B}_{0}^{n+1}\left|\sigma_{k}\right\rangle=\frac{(n+1)(k-n)}{N} \mathcal{B}_{0}^{n+1}\left|\sigma_{k}\right\rangle
$$

and this vanishes only if $n=k$.

It has already been mentioned that $\left|\mu_{k}\right\rangle=\mathcal{A}_{-1}^{N-k}\left|\sigma_{k}\right\rangle=\mathcal{B}_{0}^{k}\left|\sigma_{k}\right\rangle$. The missing highestweight conditions in (3.6), namely,

$$
\mathcal{A}_{p}\left|\mu_{k}\right\rangle=\mathcal{B}_{p}\left|\mu_{k}\right\rangle=L_{p}\left|\mu_{k}\right\rangle=0 \quad \text { for } \quad p \geq 1
$$

follow directly from (5.13).

\section{Parafermionic character formulae}

The character formula for the spin fields codes the number of independent states, level by level, with appropriate subtraction due to the presence of singular vectors. In the Virasoro, as well as in the affine $\widehat{s u}(2)$ case, this subtraction is actually an infinite process: the two primary singular vectors both have two singular vectors themselves and it turns out that they are identical; therefore, by subtracting the two primary singular vectors and their descendents, we take out too much, that is, we eliminate twice the secondary singular vectors. To correct for this, we need to add once the contribution of the secondary singular vectors. This process repeats itself constantly and that leads to a character formula expressed in terms of alternate additions and subtractions.

Exactly the same process appears here. The two primary singular vectors $\left|\chi_{N-k+1}\right\rangle$ and $\left|\chi_{k+1}^{\prime}\right\rangle$ have themselves two singular vectors each, and the two sets of secondary singular vectors coincide.

The explicit expression for the two sequences of singular vectors is as follows. Type-I, which are built from $\left|\chi_{k+1}^{\prime}\right\rangle$, start with a rightmost $\mathcal{B}$ factor:

$$
\begin{aligned}
\left|\Lambda_{k, \ell}^{\mathrm{I}(\mathrm{i})}\right\rangle & =\mathcal{B}_{0}^{2 \ell(N+2)+k+1} \mathcal{A}_{-1}^{(2 \ell-1)(N+2)+k+1} \cdots \mathcal{A}_{-1}^{(N+2)+k+1} \mathcal{B}_{0}^{k+1}\left|\sigma_{k}\right\rangle \\
\left|\Lambda_{k, \ell}^{\mathrm{I}(\mathrm{ii})}\right\rangle & =\mathcal{A}_{-1}^{(2 \ell+1)(N+2)+k+1} \mathcal{B}_{0}^{2 \ell(N+2)+k+1} \cdots \mathcal{A}_{-1}^{(N+2)+k+1} \mathcal{B}_{0}^{k+1}\left|\sigma_{k}\right\rangle
\end{aligned}
$$

that is,

$$
\left|\Lambda_{k, \ell}^{\mathrm{I}(\mathrm{i})}\right\rangle=\mathcal{B}_{0}^{2 \ell(N+2)+k+1}\left|\Lambda_{k, \ell-1}^{\mathrm{I}(\mathrm{ii})}\right\rangle, \quad\left|\Lambda_{k, \ell}^{\mathrm{I}(\mathrm{ii})}\right\rangle=\mathcal{A}_{-1}^{(2 \ell+1)(N+2)+k+1}\left|\Lambda_{k, \ell}^{\mathrm{I}(\mathrm{i})}\right\rangle
$$


while Type-II start with a rightmost $\mathcal{A}$ factor:

$$
\begin{aligned}
\left|\Lambda_{k, \ell}^{\mathrm{II}(\mathrm{i})}\right\rangle & =\mathcal{A}_{-1}^{(2 \ell+1)(N+2)-k-1} \mathcal{B}_{0}^{2 \ell(N+2)-k-1} \cdots \mathcal{B}_{0}^{2(N+2)-k-1} \mathcal{A}_{-1}^{(N+2)-k-1}\left|\sigma_{k}\right\rangle \\
\left|\Lambda_{k, \ell}^{\mathrm{II}(\mathrm{ii})}\right\rangle & =\mathcal{B}_{0}^{(2 \ell+2)(N+2)-k-1} \mathcal{A}_{-1}^{(2 \ell+1)(N+2)-k-1} \cdots \mathcal{B}_{0}^{2(N+2)-k-1} \mathcal{A}_{-1}^{(N+2)-k-1}\left|\sigma_{k}\right\rangle
\end{aligned}
$$

that is,

$$
\left|\Lambda_{k, \ell}^{\mathrm{II}(\mathrm{i})}\right\rangle=\mathcal{A}_{-1}^{(2 \ell+1)(N+2)-k-1}\left|\Lambda_{k, \ell-1}^{\mathrm{II}(\mathrm{ii})}\right\rangle, \quad\left|\Lambda_{k, \ell}^{\mathrm{II}(\mathrm{ii})}\right\rangle=\mathcal{B}_{0}^{(2 \ell+2)(N+2)-k-1}\left|\Lambda_{k, \ell}^{\mathrm{I}(\mathrm{i})}\right\rangle
$$

Notice that

$$
\left|\Lambda_{k, 0}^{\mathrm{I}(\mathrm{i})}\right\rangle=\left|\chi_{N-k+1}\right\rangle, \quad\left|\Lambda_{k, 0}^{\mathrm{II}(\mathrm{i})}\right\rangle=\left|\chi_{k+1}^{\prime}\right\rangle
$$

For both types of singular vectors, we have distinguished the cases (i) where there is an odd number of groups of terms (a group refers to a power of $\mathcal{A}_{-1}$ or $\mathcal{B}_{0}$ and will often be called a factor for short), from cases (ii) which have an even number of factors.

We first check a necessary condition for these vectors to be singular, which is that their dimension be related to their charge in exactly the same way as for the spin field.

The total charge of these vectors is given by

$$
\begin{aligned}
&\left|\Lambda_{k, \ell}^{\mathrm{I}(\mathrm{i})}\right\rangle: q=-2 \ell(N+2)-k-2 \\
&\left|\Lambda_{k, \ell}^{\mathrm{I}(\mathrm{ii})}\right\rangle: q=2(\ell+1)(N+2)+k: q \\
&\left|\Lambda_{k, \ell}^{\mathrm{II}(\mathrm{i})}\right\rangle: q=2(\ell+1)(N+2)-k-2 \\
&\left|\Lambda_{k, \ell}^{\mathrm{II}(\mathrm{ii})}\right\rangle: q=-2 \ell(N+2)+k
\end{aligned}
$$

In order to compute their dimension in a simple way, we first make a simple observation. All these singular vectors have the structure

$$
|\Lambda\rangle=\mathcal{C}^{p+1}\left|\Lambda^{\prime}\right\rangle
$$

where $\mathcal{C}$ is either $\mathcal{A}_{-1}$ or $\mathcal{B}_{0}$. It is simple to check that the sum of the fractional indices of the $p$ rightmost $\mathcal{C}$-modes add up to zero; in fact, reinserting these fractional parts, we have the following pattern, common to all cases:

$$
|\Lambda\rangle=\mathcal{C}_{(p+1) / N}\left[\mathcal{C}_{(p-1) / N} \mathcal{C}_{(p-3) / N} \cdots \mathcal{C}_{-(p-3) / N} \mathcal{C}_{-(p-1) / N}\right]\left|\Lambda^{\prime}\right\rangle
$$


That makes manifest the fact that the sum of the modes of the operators in the square bracket add up to zero. As a result, the dimension of $|\Lambda\rangle$ is simply fixed by that of the state on which the $\mathcal{C}$-string acts together with the mode of the leftmost $\mathcal{C}$ operator:

$$
h_{\Lambda}=h_{\Lambda^{\prime}}-\frac{p+1}{N}
$$

Notice finally that the mode of this leftmost operator, when multiplied by $N$, is equal to the number of $\mathcal{C}$ operators in the expression for the singular vector. In other words, we can read off the dimension of the singular vectors simply from the total sum of the various powers of $\mathcal{A}_{-1}$ or $\mathcal{B}_{0}$ factors that it contains, multiplied by $-1 / N$ :

$$
h_{\Lambda}=h_{\sigma_{k}}-\frac{1}{N}\left[\# \mathcal{A}_{-1}+\# \mathcal{B}_{0}\right]
$$

Let us then evaluate these numbers of modes in each singular vector. For type-I (i) we have $\ell+1 \mathcal{B}_{0}$-factors and $\ell \mathcal{A}_{-1}$-factors (since the type-I singular vectors start with $\mathcal{B}_{0}^{k+1}$ ); for type-I (ii), we have $\ell+1$ factors of $\mathcal{A}_{-1}$ and therefore $\ell+1 \mathcal{B}_{0}$-factors. The total number of $\mathcal{A}_{-1}$ and $\mathcal{B}_{0}$ operators in each case is respectively:

$$
\begin{array}{ll}
\text { type }-\mathrm{I}(\mathrm{i}): & \# \mathcal{A}_{-1}=r_{\ell-1} \equiv \ell[\ell(N+2)+k+1] \\
& \# \mathcal{B}_{0}=s_{\ell} \equiv(\ell+1)[(\ell(N+2)+k+1] \\
\operatorname{type}-\mathrm{I}(\mathrm{ii}): & \# \mathcal{A}_{-1}=r_{\ell}=(\ell+1)[(\ell+1)(N+2)+k+1] \\
& \# \mathcal{B}_{0}=s_{\ell}=(\ell+1)[(\ell(N+2)+k+1]
\end{array}
$$

For type-II singular vectors, the analogous results are

$$
\begin{array}{ll}
\text { type }- \text { II (i) : } & \left.\# \mathcal{A}_{-1}=r_{\ell}^{\prime} \equiv(\ell+1)\right)[(\ell+1)(N+2)-k-1] \\
& \# \mathcal{B}_{0}=s_{\ell-1}^{\prime} \equiv \ell[(\ell+1)(N+2)-k-1] \\
\text { type }- \text { II (ii) : } & \# \mathcal{A}_{-1}=r_{\ell}^{\prime}=(\ell+1)[(\ell+1)(N+2)-k-1] \\
& \# \mathcal{B}_{0}=s_{\ell}^{\prime}=(\ell+1)[(\ell+2)(N+2)-k-1]
\end{array}
$$

We can thus read off the dimensions of the singular vectors from (6.10), (6.11) and (6.12). In all cases, we can verify that the dimension $h$ and the charge $q$ are related by

$$
h=\frac{q(N-q)}{2 N(N+2)}
$$

as it should for a highest-weight state. 
But having made this observation, we have essentially established that these are genuine singular vectors since the nontrivial part of the proof amounts to show that $\left|\Lambda_{k, \ell}^{\mathrm{I}(\mathrm{i})}\right\rangle$ and $\left|\Lambda_{k, \ell}^{\mathrm{II}(\mathrm{ii})}\right\rangle$ are annihilated by $\mathcal{A}_{0}$ while $\left|\Lambda_{k, \ell}^{\mathrm{I}(\mathrm{ii})}\right\rangle$ and $\left|\Lambda_{k, \ell}^{\mathrm{II}(\mathrm{i})}\right\rangle$ are annihilated by $\mathcal{B}_{1}$. But this reduces to a computation equivalent to (5.10) and that simply forces a relation between the dimension of the vector and its charge (both parametrized by $n$ ), which is precisely the relation (6.13) already established.

In principle, we have only half of the explicit expressions of the singular vectors. We claim that each singular vector has itself two primary singular vectors, but we have only displayed one of them. Indeed, consider for instance:

$$
\left|\Lambda_{k, 0}^{\mathrm{I}(\mathrm{i})}\right\rangle=\mathcal{B}_{0}^{k+1}\left|\sigma_{k}\right\rangle \equiv\left|\sigma_{-k-2}\right\rangle
$$

where we have defined a spin state with a charge outside of the 'Kac table'. If we extend directly the expression for the $\left|\sigma_{k}\right\rangle$ singular vectors to cases where $k$ lies outside the range $0 \leq k \leq N-1$, we would have the following two primary singular vectors:

$$
\mathcal{A}_{-1}^{N+2-(-k-2)-1}\left|\sigma_{-k-2}\right\rangle=\mathcal{A}_{-1}^{N+2+k+1} \mathcal{B}_{0}^{k+1}\left|\sigma_{k}\right\rangle
$$

and

$$
\mathcal{B}_{0}^{(-k-2)+1}\left|\sigma_{-k-2}\right\rangle=\mathcal{B}_{0}^{-k-1} \mathcal{B}_{0}^{k+1}\left|\sigma_{k}\right\rangle=\left|\sigma_{k}\right\rangle
$$

In the second case we simply return to the highest-weight state of the previous layer, while the first one yields a genuine new singular vector. Actually, the expression of the other primary singular vector does not have a simple factorizable expression when written from $\left|\sigma_{-k-2}\right\rangle$. However, we argue below that the two singular vectors at one layer - these have the same value of $\ell$ and are of the same kind (i) or (ii), - have common primary singular vectors exactly as in the Virasoro case. Granting this, we have then the expression of all the singular vectors, starting from the highest-weight state.

Given the close relation between the parafermionic theory and the $\widehat{s u}(2)_{N}$ model, the above expressions appear to be rather natural a posteriori. Indeed, the $\widehat{s u}(2)_{N}$ generators can be expressed in terms of the parafermionic field and a single boson $\varphi$ (with OPE $\varphi(z) \varphi(w) \sim-\ln (z-w))$ as follows:

$$
\begin{aligned}
J^{\dagger}(z) & =\sqrt{N} \psi_{1}(z) e^{i \sqrt{2 / N} \varphi(z)} \\
J^{-}(z) & =\sqrt{N} \psi_{1}^{\dagger}(z) e^{-i \sqrt{2 / N} \varphi(z)} \\
J^{0}(z) & =i \sqrt{2 N} \partial \varphi(z)
\end{aligned}
$$


The label $k$ of the spin field corresponds to the finite Dynkin label of the affine weight $\hat{\lambda}$ at level $N: \hat{\lambda}=[N-k, k]$. In the Chevalley basis where $J_{0}^{-}=f_{1}, J_{-1}^{\dagger}=f_{0}$ and $J_{0}^{0}=h_{1} / 2$, with the action of the $h_{i}$ being defined as

$$
h_{i}|\hat{\lambda}\rangle=\lambda_{i}|\hat{\lambda}\rangle \quad \text { with } \quad \lambda_{0}=N-k, \quad \lambda_{1}=k
$$

the sequence of affine singular vectors take the following compact form

$$
f_{i+j}^{h_{i+j}+1} \cdots f_{i+1}^{h_{i+1}+1} f_{i}^{h_{i}+1}|\hat{\lambda}\rangle
$$

where

$$
f_{i}^{h_{i}+1}|\hat{\lambda}\rangle \equiv f_{i}^{\lambda_{i}+1}|\hat{\lambda}\rangle
$$

starting with $i=0$ or 1 and with the addition of indices defined modulo 2. Since there are no singular vectors in the $\widehat{u}(1)$ sector, the structure of these vectors is directly transferred into the parafermionic theory, with $f_{0}=J_{-1}^{\dagger} \propto \mathcal{A}_{-1}$ and $f_{1}=J_{0}^{-} \propto \mathcal{B}_{0}$. But that provides us with the missing piece of the previous argument: the embedding pattern of the $\widehat{s u}(2)_{N}$ theory is directly transposed into the parafermionic theory.

Unfortunately, the last step of the argument relies on the coset description and it is thus not intrinsically parafermionic. To partly supply for this, we will exemplify below this embedding argument with few illustrative examples pertaining to the Ising model.

How do we evaluate the effect of these charged singular vectors, say in a module with zero relative charge? Clearly, we have to 'fold' each singular vector by the action of an appropriate number of $\mathcal{A}_{-1}$ or $\mathcal{B}_{0}$ factors to render the total relative charge zero. Therefore, if the singular vector ends with a $\mathcal{A}_{-1}$ factor, we need to act on it with the appropriate number of $\mathcal{B}_{0}$ factors required to make its relative charge zero; the fact that we act with $\mathcal{B}_{0}$ factors ensures that we will get the lowest level at which a zero relative charge descendent appears. Since the resulting state is obtained by the actions of $\mathcal{A}_{-1}$ and $\mathcal{B}_{0}$ modes, this level is simply the number of $\mathcal{A}_{-1}$ factors already contained in the expression of the singular vector. On the other hand, if the singular vector ends with a $\mathcal{B}_{0}$ factor, we need to compensate with the action of the appropriate number of $\mathcal{A}_{-1}$ modes to get the lowest zero relative charge descendent: but the total number of $\mathcal{A}_{-1}$ modes in the resulting state is simply the total number of $\mathcal{B}_{0}$ modes already present in the original singular vector. 
The expression for the levels at which the folding of the singular vectors does occur is thus given by (6.11) and (6.12).

To build up the character formula for $\sigma_{k}$, we need to subtract the singular vectors of type (i) and add those of type (ii). Denote by $V_{k, q}$ the free module of relative charge $2 q$ over $\left|\sigma_{k}\right\rangle$ and by $V_{k, q, \ell}^{\mathrm{J}}$ the free module of relative charge $2 q$ over $\left|\Lambda_{k, \ell}^{\mathrm{J}}\right\rangle$. The $\left|\sigma_{k}\right\rangle$ irreducible module $M_{k, q}$ takes thus the following form

$$
M_{k, q}=V_{k, q}-\sum_{\ell=0}^{\infty}\left[V_{k, q, \ell}^{\mathrm{II}(\mathrm{i})}+V_{k, q, \ell}^{\mathrm{II}(\mathrm{i})}\right]+\sum_{\ell=0}^{\infty}\left[V_{k, q, \ell}^{\mathrm{I}(\mathrm{ii})}+V_{k, q, \ell}^{\mathrm{II}(\mathrm{ii})}\right]
$$

For free modules, the counting of states, level by level, is given by eq. (4.20) with $r=q \geq 0$. This is our main result. We now make it more explicit.

The character of an uncharged $(q=0)$ module for the spin state $\sigma_{k}$ is thus given by

$$
\chi_{k, 0}=q^{h_{\sigma_{k}}-c / 24} \sum_{s=0} q^{s} g_{k, 0}(s)
$$

where $g_{k, 0}(s)$ corresponds to the number of states at level $s$ and it is given by

$$
g_{k, 0}(s)=\sum_{\ell=0}^{\infty} g_{k, 0, \ell}(s)
$$

with

$$
g_{k, 0, \ell}(s)=G_{0,0} \delta_{\ell, 0}-G_{r_{\ell-1}, s_{\ell}}-G_{r_{\ell}^{\prime}, s_{\ell-1}^{\prime}}+G_{r_{\ell}, s_{\ell}}+G_{r_{\ell}^{\prime}, s_{\ell}^{\prime}}
$$

where

$$
G_{a, b}(s) \equiv \sum_{j=0}^{s} \sum_{s_{1}=j}^{s} p^{(j-a)}\left(s_{1}-j\right) p^{(j-b)}\left(s-s_{1}\right)
$$

with $a, b$ two non-negative integers. 目 Notice that

$$
G_{a, b}(s)=0 \quad \text { if } \quad s<\max (a, b)
$$

For instance, for the Ising model, $N=2$, with $k=0$, this reproduces the vacuum Virasoro character (1.1) while for $k=1$, this is the spin character also written in (1.1). Still another character formula for these two fields is given by the usual Rocha-Caridi expression [12] built from a Virasoro module. We stress however that the subtraction of the Virasoro

5 The $k$-dependence of $G$ is hidden in $r_{\ell}, s_{\ell}$ and their prime versions. 
singular vectors occur at levels that do not match the levels at which parafermionic singular vectors arise.

Let us work out the multiplicities of the first few levels of the vacuum Ising character as expressed in the parafermionic language. Up to level 8, it takes the form:

$$
q^{1 / 48} \chi_{1,1}=\sum_{s=0}^{\infty} q^{s}\left[G_{0,0}-G_{0,1}-G_{3,0}+G_{5,1}+G_{3,7}-\cdots\right]
$$

Let us list the number of states level by level associated to each of these factors, or equivalently, evaluate these expressions $G_{a, b}(s)$. We have

$\begin{array}{llllll} & G_{0,0} & G_{0,1} & G_{3,0} & G_{5,1} & G_{3,7} \\ s=0: & 1 & 0 & 0 & 0 & 0 \\ s=1: & 1 & 1 & 0 & 0 & 0 \\ s=2: & 3 & 2 & 0 & 0 & 0 \\ s=3: & 6 & 4 & 1 & 0 & 0 \\ s=4: & 12 & 8 & 2 & 0 & 0 \\ s=5: & 21 & 15 & 5 & 1 & 0 \\ s=6: & 38 & 27 & 10 & 2 & 0 \\ s=7: & 63 & 47 & 19 & 5 & 1 \\ s=8: & 106 & 79 & 34 & 10 & 2\end{array}$

The number of states, up to level 8 is then

$$
\begin{aligned}
1 & +q(1-1)+q^{2}(3-2)+q^{3}(6-4-1)+q^{4}(12-8-2) \\
& +q^{5}(21-15-5+1)+q^{6}(38-27-10+2) \\
& +q^{7}(63-47-19+5+1)+q^{8}(106-79-34+10+2)+\cdots \\
& =1+q^{2}+q^{3}+2 q^{4}+2 q^{5}+3 q^{6}+3 q^{7}+5 q^{8}+\cdots
\end{aligned}
$$

The $q$ expansion of the character (6.27) (as well as that of the spin field) has been checked up to level 85 .

Let us make use of this example to illustrate the embedding of singular vectors. The first two singular vectors (the primary ones) of the Ising vacuum are

$$
\mathcal{B}_{0}|0\rangle \quad \text { and } \quad \mathcal{A}_{-1}^{3}|0\rangle
$$


and their descendents are respectively

$$
\mathcal{A}_{-1}^{5} \mathcal{B}_{0}|0\rangle \quad \text { and } \quad \mathcal{B}_{0}^{7} \mathcal{A}_{-1}^{3}|0\rangle
$$

Now is $\mathcal{A}_{-1}^{5} \mathcal{B}_{0}|0\rangle$ a descendent of $\mathcal{A}_{-1}^{3}|0\rangle$ ? To see this, consider the reexpression of $\mathcal{A}_{-1}^{5} \mathcal{B}_{0}|0\rangle$ in terms of basis states for which all $\mathcal{A}$ operators are at the right (which differs thus from the basis considered previously). The independent states at level 5 and charge 8 with the $\mathcal{A}$ ordered at the right are $\mathcal{B}_{0} \mathcal{A}_{-1}^{5}|0\rangle$ and $\mathcal{A}_{-2} \mathcal{A}_{-1}^{3}|0\rangle$. Therefore, $\mathcal{A}_{-1}^{5} \mathcal{B}_{0}|0\rangle$ is necessarily a linear combination of these two states. But both states are manifestly descendents of $\mathcal{A}_{-1}^{3}|0\rangle$. Similarly, it is simple to see that $\mathcal{B}_{0}^{7} \mathcal{A}_{-1}^{3}|0\rangle$ is a descendent of $\mathcal{B}_{0}|0\rangle$ : the list of all states at level 3 and total charge -8 with $\mathcal{B}$ operators ordered at the right is

$$
\begin{array}{llll}
\mathcal{A}_{-1}^{3} \mathcal{B}_{0}^{7}|0\rangle, & \mathcal{A}_{-2} \mathcal{A}_{-1} \mathcal{B}_{0}^{6}|0\rangle, & \mathcal{A}_{-1}^{2} \mathcal{B}_{-1} \mathcal{B}_{0}^{5}|0\rangle, \quad \mathcal{A}_{-1} \mathcal{B}_{-2} \mathcal{B}_{0}^{4}|0\rangle, \quad \mathcal{A}_{-2} \mathcal{B}_{-1} \mathcal{B}_{0}^{4}|0\rangle, \\
\mathcal{A}_{-3} \mathcal{B}_{0}^{5}|0\rangle, & \mathcal{A}_{-1} \mathcal{B}_{-1}^{2} \mathcal{B}_{0}^{3}|0\rangle, & \mathcal{B}_{-3} \mathcal{B}_{0}^{3}|0\rangle, & \mathcal{B}_{-2} \mathcal{B}_{-1} \mathcal{B}_{0}^{2}|0\rangle,
\end{array}
$$

(and indeed $G_{0,4}(s=3)=10$ ) and all these states have a rightmost factor $\mathcal{B}_{0}|0\rangle$ i.e., they are descendents of $\mathcal{B}_{0}|0\rangle$.

For modules of relative charge $2 t>0$, we simply replace all

$$
G_{a, b} \quad \rightarrow \quad G_{a, b+t}
$$

and multiply the prefactor in the character expression (6.23) by $q^{\Delta}$ with $\Delta$, instead of being $h_{\sigma_{k}}-c / 24$ is given by:

$$
\Delta=h_{\varphi_{k}^{(t)}}-c / 24-|t|
$$

(the absolute value is unnecessary but it makes the formula valid in the case where $t$ is negative as indicated below). The factor $q^{-t}$ is introduced in order to reshuffle the level counting from the state associated to $\varphi_{k}^{(t)}$, itself at level $t$ below the highest-weight state $\left|\sigma_{k}\right\rangle$. If $2 t<0$, we have the following modifications: $G_{a, b} \rightarrow G_{a-|t|, b}$ and $q^{h_{\sigma_{k}}-c / 24} \rightarrow q^{\Delta}$, with $\Delta$ defined in $(6.34)$.

Let us work out the fermion character of the Ising model. The fermion is expressed as $|\psi\rangle=\mathcal{A}_{-1}|0\rangle$. Its character is computed as follows: we list all the charge 2 descendents of the vacuum and subtract the contribution of the charge -4 descendent of the singular 
vector $\mathcal{A}_{-1}|0\rangle$ and the charge 4 descendent of the singular vector $\mathcal{B}_{0}|0\rangle$, etc. The explicit form of the first few charge 2 descendents of the vacuum are

$$
\begin{array}{ll}
s=1: & \mathcal{A}_{-1}|0\rangle \\
s=2: & \mathcal{A}_{-2}|0\rangle, \mathcal{A}_{-1}^{2} \mathcal{B}_{0}|0\rangle \\
s=3: & \mathcal{A}_{-3}|0\rangle, \mathcal{A}_{-2} \mathcal{A}_{-1} \mathcal{B}_{0}|0\rangle, \mathcal{A}_{-1}^{2} \mathcal{B}_{-1}|0\rangle, \mathcal{A}_{-1}^{3} \mathcal{B}_{0}^{2}|0\rangle
\end{array}
$$

However, when these states are counted from the fermion state $\mathcal{A}_{-1}|0\rangle$, the above values of $s$ must be reduced by 1 . Up to level 5 , the fermion character reads then:

$$
q^{-1 / 2+1 / 48} \chi_{2,1}=G_{0,1}-G_{0,2}-G_{3,1}+G_{5,2}+-\cdots
$$

The values of these $G_{j^{\prime}, j^{\prime \prime}}$ at each level are:

$\begin{array}{lllll} & G_{0,1} & G_{0,2} & G_{3,1} & G_{5,2} \\ s-1=0: & 1 & 0 & 0 & 0 \\ s-1=1: & 2 & 1 & 0 & 0 \\ s-1=2: & 4 & 2 & 1 & 0 \\ s-1=3: & 8 & 5 & 2 & 0 \\ s-1=4: & 15 & 9 & 5 & 1 \\ s-1=5: & 27 & 18 & 9 & 2\end{array}$

The first few terms in the development of the fermion character are then

$$
\begin{aligned}
1 & +q(2-1)+q^{2}(4-2-1)+q^{3}(8-5-2) \\
& +q^{4}(15-9-5+1)+q^{5}(27-18-9+2) \\
& =1+q+q^{2}+q^{3}+2 q^{4}+2 q^{5}+\cdots
\end{aligned}
$$

which is to be compared with the $q$-expansion of $\chi_{2,1}$ in (1.1). Again this has been verified up to level 85 by computer.

The various characters for the three-state Potts model have also been extensively checked.

Finally, we stress that the parafermionic characters just obtained provide new expressions for the $\widehat{s u}(2)$ string functions [13]. Indeed, the character of $\sigma_{k}$ with relative charge $2 \ell$, with $\ell=k-m$ is simply the normalized string function $c_{m}^{k}$ for the weight $m \omega_{1}$ in the affine representation $\hat{\lambda}=[N-k, k]$ (see [6, 8] and sect. 18.6 of [10]). 


\section{Conclusion}

In this work we have deepened some aspects of the representation theory of the parafermionic conformal models. It leads in particular to new parafermionic character formulae and thereby, to new expressions for $\widehat{s u}(2)_{N}$ string functions. These new formulae may have interesting combinatorial descriptions which may give hints for their direct proof.

We should also point out that in [14], character formulae have also been worked out directly from the $Z_{V}$ algebra introduced in [15] and which is equivalent to the parafermionic algebra. However, the resulting characters are fermionic in structure (in the terminology of [16] - i.e., these are written in product form). Equalling the bosonic-type formulae obtained here and these fermionic characters provides further interesting identities whose direct verification would remain an interesting problem.

In relation to the motivation formulated in the introduction, it is fair to say that building the characters directly from the parafermionic modules has lead us to the most complicated character expressions that have been found so far for the parafermionic fields. Evenmore, the Ising case does not provide notable simplifications: actually, it captures the essential structure of the generic case. It certainly indicates that the rich off-critical Ising results will not have a straightforward extension to the class of parafermionic models.

On the other hand, as pointed out also in the introduction, the techniques elaborated here are expected to be applicable to more general parafermionic models for which there may be no alternative formulations in terms of known conformal field theories. Moreover, the results of this work should allow us to reconsider, from a new point of view, the classification of the integrable perturbations of the parafermionic models, for instance, using the singular-vector argument of [17]. That will undoubtedly require the extension of the present theories to the non-unitary sector. We hope to report on these questions elsewhere.

\section{Appendix A. Proofs related to the basis of independent states}

We first prove the first part of lemma 1, that is, that on a generic highest-weight state $\left|\phi_{q}\right\rangle$, any state at fixed level $s$, built out of $j$ operators $\mathcal{A}$, can be expressed as a linear

combination of terms of the form $\mathcal{A}_{-n_{1}} \mathcal{A}_{-n_{2}} \ldots \mathcal{A}_{-n_{j}}\left|\phi_{q}\right\rangle$ with all $n_{i} \geq 1$ (and of course 
$\sum_{i} n_{i}=s \geq j$ ). We thus want to show that, by means of the commutation relations, we can always transform a term containing a positive index into a combination of terms with all indices negative. Consider then, say, a term of the type

$$
\mathcal{A}_{-n_{1}} \mathcal{A}_{n_{2}} \ldots \mathcal{A}_{-n_{j}}\left|\phi_{q}\right\rangle
$$

(we suppose in this and in the following expressions that all the $n_{i}$ are strictly positive). Using the commutation relations (2.13), we can rewrite it under the form

$$
\sum_{\ell=0}^{\infty}\left[-c_{\ell+1} \mathcal{A}_{-n_{1}} \mathcal{A}_{n_{2}-\ell-1} \mathcal{A}_{-n_{3}+\ell+1} \ldots \mathcal{A}_{-n_{j}}+c_{\ell} \mathcal{A}_{-n_{1}} \mathcal{A}_{-n_{3}-\ell} \mathcal{A}_{n_{2}+\ell \ldots \mathcal{A}_{-n_{j}}}\right]\left|\phi_{q}\right\rangle
$$

(only the indices of the second and third modes from the left are affected). The effect is to increase the indices toward the right: after a certain number of iterations, the index of the second mode can be made strictly negative. The resulting states either have all their indices negative or there are terms whose third mode has a positive index. But then we repeat the procedure with the second and third modes replaced by the third and the fourth ones, and repeat again the whole process until we hit the last pair of modes; then, because the rightmost mode acts on a highest-weight state, we can drop all the terms for which this last mode has a positive index and we are left with only those terms which all have strictly negative modes.

The second part of the lemma states that products of $\mathcal{A}$ modes can always be reorganized such that $n_{k} \geq n_{l}$ if $k<l$. To prove this, consider a term of the form $\mathcal{A}_{-n_{1}} \mathcal{A}_{-n_{2}} \mathcal{A}_{-n_{3}} \ldots \mathcal{A}_{-n_{j}}\left|\phi_{q}\right\rangle$ with say $n_{2}<n_{3}$, with all other terms ordered as expected (that is, for which $n_{k} \geq n_{l}$ if $k<l$ ). We again use the commutation relations (2.13) to write it as

$$
\sum_{\ell=0}^{\infty}\left[-c_{\ell+1} \mathcal{A}_{-n_{1}} \mathcal{A}_{-n_{2}-\ell-1} \mathcal{A}_{-n_{3}+\ell+1} \ldots \mathcal{A}_{-n_{j}}+c_{\ell} \mathcal{A}_{-n_{1}} \mathcal{A}_{-n_{3}-\ell} \mathcal{A}_{-n_{2}+\ell \ldots \mathcal{A}_{-n_{j}}}\right]\left|\phi_{q}\right\rangle
$$

Hence, we have reexpressed our term into a sum of terms for which the second mode has a larger absolute value (and, accordingly, the third mode has a lower absolute value). Reapplying the argument, we can rewrite all terms into a combination of terms of the type $\mathcal{A}_{-n_{1}} \mathcal{A}_{-n_{2}^{\prime}} \mathcal{A}_{-n_{3}^{\prime}} \ldots \mathcal{A}_{-n_{j}}\left|\phi_{q}\right\rangle$ for which the condition $n_{2}^{\prime} \geq n_{3}^{\prime}$ is satisfied. However, the ordering of the first and second modes or the third and the fourth modes may no longer satisfy the initial ordering prescription, but we then repeat the procedure for each pair of 
contiguous indices that are not in proper order until all indices are non-decreasing toward the right.

The analysis presented for the $\mathcal{A}$ strings applies directly for the $\mathcal{B}$ strings, the only modification being that, in this case, the indices can also be zero.

Consider now mixed states. We want to show that we can order separately the $\mathcal{A}$ strings and the $\mathcal{B}$ strings but that there is no mixed ordering. That all positive indices can be eliminated is rather clear and does not depend upon the mixed nature of the string of operators. It is also clear that we can move all the $\mathcal{B}$ operators to the right and then use lemma 2 to order their indices. However, this ordering is not dependent upon the fact that the string of $\mathcal{B}$ operators acts on a highest-weight state: as a result, we can order the $\mathcal{A}$ modes in the same way. In other words, as far as the characterization of states is concerned, that $\mathcal{A}$ modes act on a highest-weight state or that a $\mathcal{B}$-string is inserted in between does not matter. In that regard, a positive mode does not annihilate the $\mathcal{B}$-string but it produces states already present, that is, already having the proper ordering.

\section{Appendix B. From parafermionic to Virasoro singular vectors for the Ising and the three-state Potts model}

We have shown that the singular states of the highest-weight module of $\left|\sigma_{k}\right\rangle$ are necessarily charged. There are obviously uncharged (relative to $\left|\sigma_{k}\right\rangle$ ) descendents of these singular vectors. For instance, the lowest descendent of the vacuum $\left(|0\rangle=\left|\sigma_{0}\right\rangle\right)$ singular vector $\mathcal{B}_{0}|0\rangle$ is obtained by acting with $\mathcal{A}_{-1}$ :

$$
\mathcal{A}_{-1} \mathcal{B}_{0}|0\rangle=\frac{N+2}{N} L_{-1}|0\rangle
$$

as expected. Uncharged descendents of singular vectors at level two arise in the module of $\left|\sigma_{k}\right\rangle$ for $k=1$ or $k=N-1$. Let us work out their explicit form, writing them with as much Virasoro modes as possible. The descendent of relative charge 4 and level two of $\left|\chi_{2}\right\rangle=\mathcal{B}_{0}^{2}\left|\sigma_{1}\right\rangle$ is unique:

$$
\mathcal{A}_{-1}^{2} \mathcal{B}_{0}^{2}\left|\sigma_{1}\right\rangle=-\frac{N+2}{N^{2}}\left[L_{-2}-(N+2) L_{-1}^{2}+N \mathcal{A}_{-2} \mathcal{B}_{0}\right]\left|\sigma_{1}\right\rangle
$$


(Uniqueness is obvious, e.g., we cannot act with say $\mathcal{A}_{-2} \mathcal{A}_{0}$ since $\mathcal{A}_{0} \mathcal{B}_{0}^{2}\left|\sigma_{1}\right\rangle=0$ by (5.13).) On the other hand, the descendent of relative charge -4 and level two of $\left|\chi_{2}^{\prime}\right\rangle=\mathcal{A}_{-1}^{2}\left|\sigma_{N-1}\right\rangle$ is

$$
\mathcal{B}_{0}^{2} \mathcal{A}_{-1}^{2}\left|\sigma_{N-1}\right\rangle=-\frac{(N+2)(N+3)}{N^{2}}\left[L_{-2}-\frac{N+2}{N+3} L_{-1}^{2}-\frac{N}{N+3} \mathcal{A}_{-2} \mathcal{B}_{0}\right]\left|\sigma_{N-1}\right\rangle
$$

In a similar way, we can compute the level-three descendents of the singular vectors $\left|\chi_{3}\right\rangle$ and $\left|\chi_{3}^{\prime}\right\rangle$, of relative charge \pm 6 respectively; they are $\mathcal{A}_{-1}^{3} \mathcal{B}_{0}^{3}\left|\sigma_{2}\right\rangle$, which is proportional to

$$
\begin{aligned}
& {\left[L_{-1} L_{-2}-\frac{N+2}{4} L_{-1}^{3}-\frac{N+4}{2(N+2)} L_{-3}-\frac{7 N+4}{2(N+2)} \mathcal{A}_{-3} \mathcal{B}_{0}\right.} \\
& \left.+\frac{N(N+6)}{4(N+2)} L_{-1} \mathcal{A}_{-2} \mathcal{B}_{0}+\frac{N^{2}}{2(N+2)} \mathcal{A}_{-2} \mathcal{B}_{0} L_{-1}\right]\left|\sigma_{2}\right\rangle
\end{aligned}
$$

and $\mathcal{B}_{0}^{3} \mathcal{A}_{-1}^{3}\left|\sigma_{N-2}\right\rangle$, proportional to

$$
\begin{aligned}
& {\left[L_{-1} L_{-2}-\frac{N+2}{3 N+10} L_{-1}^{3}-\frac{4\left(N^{2}+6 N+9\right)}{(3 N+10)(N+2))} L_{-3}\right.} \\
& -\frac{4\left(3 N^{2}+16 N+10\right)}{\left(N^{2}-4\right)(3 N+10)} \mathcal{A}_{-3} \mathcal{B}_{0}+\frac{\left.N\left(N^{2}+10 N+12\right)\right)}{\left(3 N^{2}+4 N-20\right)(N+2)} L_{-1} \mathcal{A}_{-2} \mathcal{B}_{0} \\
& \left.-\frac{2 N^{2}(2 N+5)}{\left(3 N^{2}+4 N-20\right)(N+2)} \mathcal{A}_{-2} \mathcal{B}_{0} L_{-1}\right]\left|\sigma_{N-2}\right\rangle
\end{aligned}
$$

Let us now look at the relation between these vectors and the usual Virasoro singular vectors of the Ising model. In that case $N=2$, so that $\left|\sigma_{N-1}\right\rangle$ reduces to $\left|\sigma_{1}\right\rangle$. We have thus two expressions (B.2) and (B.3) for the descendents of the parafermionic singular vector in the vacuum module:

$$
\begin{aligned}
& {\left[L_{-2}-4 L_{-1}^{2}+2 \mathcal{A}_{-2} \mathcal{B}_{0}\right]\left|\sigma_{1}\right\rangle=0} \\
& {\left[5 L_{-2}-4 L_{-1}^{2}-2 \mathcal{A}_{-2} \mathcal{B}_{0}\right]\left|\sigma_{1}\right\rangle=0}
\end{aligned}
$$

Eliminating $\mathcal{A}_{-2} \mathcal{B}_{0}\left|\sigma_{1}\right\rangle$ between these two equations yields

$$
\left[L_{-2}-\frac{4}{3} L_{-1}^{2}\right]\left|\sigma_{1}\right\rangle=0
$$

which is the expected expression for the level-two singular vector of the Ising spin field $\phi_{1,2}$. Consider next the level-two and level-three singular vectors of the fermion field $\phi_{1,3}=\phi_{2,1}$. Its parafermionic definition is $\mathcal{A}_{-1}|0\rangle$. The descendents of the vacuum singular vectors 
must then be folded in the charge 2 sector. The appropriate folding of $\mathcal{A}_{-1}^{3}|0\rangle$ at the lowest possible level is obtained from two applications of $\mathcal{B}_{0}$ :

$$
\mathcal{B}_{0}^{2} \mathcal{A}_{-1}^{3}|0\rangle=\left[\frac{5}{2} L_{-1}^{2} \mathcal{A}_{-1}-2 L_{-2} \mathcal{A}_{-1}-2 \mathcal{B}_{-1} \mathcal{A}_{-1}^{2}\right]|0\rangle
$$

while the appropriate folding of $\mathcal{B}_{0}|0\rangle$ at level 3 is

$$
\mathcal{A}_{-2} \mathcal{A}_{-1} \mathcal{B}_{0}|0\rangle=\left[-L_{-1}^{2} \mathcal{A}_{-1}+2 L_{-2} \mathcal{A}_{-1}-\mathcal{B}_{-1} \mathcal{A}_{-1}^{2}\right]|0\rangle
$$

The elimination of the terms $\mathcal{B}_{-1} \mathcal{A}_{-1}^{2}|0\rangle$ leads to

$$
\left[L_{-2}-\frac{3}{4} L_{-1}^{2}\right] \mathcal{A}_{-1}|0\rangle=0
$$

as it should, since $\mathcal{A}_{-1}|0\rangle=\left|\phi_{2,1}\right\rangle=|\psi\rangle$. To obtain the level-three singular vector, we compute the following descendents:

$$
\begin{aligned}
& L_{-1} \mathcal{A}_{-2} \mathcal{A}_{-1} \mathcal{B}_{0}|0\rangle=\left[2 L_{-3} \mathcal{A}_{-1}+2 L_{-2} L_{-1} \mathcal{A}_{-1}-L_{-1}^{3} \mathcal{A}_{-1}-3 \mathcal{B}_{-2} \mathcal{A}_{-1}^{2}-2 \mathcal{B}_{-1} \mathcal{A}_{-2} \mathcal{A}_{-1}\right]|0\rangle \\
& \mathcal{A}_{-3} \mathcal{A}_{-1} \mathcal{B}_{0}|0\rangle=\left[2 L_{-3} \mathcal{A}_{-1}-\frac{1}{3} L_{-1}^{3} \mathcal{A}_{-1}-2 \mathcal{B}_{-2} \mathcal{A}_{-1}^{2}-\mathcal{B}_{-1} \mathcal{A}_{-2} \mathcal{A}_{-1}\right]|0\rangle \\
& \mathcal{B}_{-1} \mathcal{B}_{0} \mathcal{A}_{-1}^{3}|0\rangle=\left[4 L_{-3} \mathcal{A}_{-1}+4 L_{-2} L_{-1} \mathcal{A}_{-1}-2 L_{-1}^{3} \mathcal{A}_{-1}-6 \mathcal{B}_{-2} \mathcal{A}_{-1}^{2}-\mathcal{B}_{-1} \mathcal{A}_{-2} \mathcal{A}_{-1}\right]|0\rangle
\end{aligned}
$$

From these three relations, we can eliminate all the terms that cannot be written as Virasoro modes acting on $\mathcal{A}_{-1}|0\rangle$ : this leads to

$$
\left[L_{-3}-2 L_{-2} L_{-1}+\frac{1}{2} L_{-1}^{3}\right] \mathcal{A}_{-1}|0\rangle=\left[L_{-3}-2 L_{-2} L_{-1}+\frac{1}{2} L_{-1}^{3}\right]|\psi\rangle
$$

which differs from the usual singular vector expression

$$
\left[L_{-3}-4 L_{-2} L_{-1}+\frac{4}{3} L_{-1}^{3}\right]|\psi\rangle
$$

only by a term proportional to the descendent of the fermionic level-two singular vector.

Let us now consider one example associated to the three-state Potts model, for which $N=3$. The lowest-level Virasoro singular vector is that of the parafermion $\psi_{1}$ which corresponds to the Virasoro field $\phi_{1,3}$. Its parafermionic description is $\mathcal{A}_{-1}|0\rangle$. The singular vectors that need to be considered are thus those of the vacuum: $\mathcal{B}_{0}|0\rangle$ and $\mathcal{A}_{-1}^{4}|0\rangle$. The appropriate descendents are:

$$
\mathcal{A}_{-2} \mathcal{A}_{-2} \mathcal{B}_{0}|0\rangle, \quad \mathcal{A}_{-3} \mathcal{A}_{-2} \mathcal{B}_{0}|0\rangle, \quad \mathcal{B}_{0}^{3} \mathcal{A}_{-1}^{4}|0\rangle
$$


and these generates linear combinations of the form:

$$
\left[a_{1} L_{-3}+a_{2} L_{-1}^{3}+a_{3} \mathcal{B}_{-1} \mathcal{A}_{-2}+a_{4} \mathcal{B}_{-2} \mathcal{A}_{-1}\right] \mathcal{A}_{-1}|0\rangle
$$

The elimination of the two terms with parafermionic modes yields the correct singular vector

$$
\left[L_{-3}-\frac{3}{4} L_{-1} L_{-2}+\frac{9}{40} L_{-1}^{3}\right]\left|\psi_{1}\right\rangle
$$

Clearly, the spin-field singular vectors of the three-state Potts model can be obtained by the same procedure.

\section{Appendix C. Recovering the free-fermion limit}

The whole structure of the parafermionic algebra and the corresponding highest-weight modules do not manifestly degenerate into a theory of free fermions as $N=2$. It is thus of interest to work out explicitly the free-fermionic limit of some of the key results obtained in a general context. At first, we will show how the parafermionic conformal algebra reduces to that of a free fermion and codes the expression of the energy-momentum tensor.

When $N=2, \psi_{1}=\psi_{1}^{\dagger}$, i.e., there is a reality condition. Hence there is no concept of charge. More precisely, the charge of a state boils down to its sector, Neveu-Schwartz (NS) or Ramond (R). Therefore, we should not distinguish the $\mathcal{A}$ modes from the $\mathcal{B}$ ones and we will denote them collectively as $b$, with half-integer (integer) modes in the NS (R) sector respectively. Consider first (2.13). When $N=2$, the coefficient $c_{\ell}^{\prime}$ becomes:

$$
c_{\ell}^{\prime}=\frac{\Gamma(\ell-1)}{\ell ! \Gamma(-1)}
$$

which implies that

$$
c_{0}^{\prime}=1, \quad c_{1}^{\prime}=-1, \quad c_{\ell>1}^{\prime}=0
$$

Consider for instance the case $q=0$ : the relation $(2.13)$ reduces to

$$
\left[\left(b_{3 / 2+n} b_{1 / 2+m}+b_{1 / 2+m} b_{3 / 2+n}\right)-\left(b_{3 / 2+m} b_{1 / 2+n}+b_{1 / 2+n} b_{3 / 2+m}\right)\right]\left|\phi_{0}\right\rangle
$$

Similar conditions are obtained in the $q=1$ sector. This shows that the anticommutator $\left\{b_{p}, b_{r}\right\}$ is necessarily a function of the sum of the indices $p+r$. To fix this function, we need to consider the other commutation relation $(2.10)$. 
We first observe that when $N=2$

$$
c_{\ell}=\ell+1
$$

Let the two sides of (2.10) act, not on a general state $\left|\phi_{q}\right\rangle$, but on the vacuum state $|0\rangle$, which is a parafermionic highest-weight state. For $n=-1$ and $m=0,(2.10)$ reduces to

$$
b_{-1 / 2} b_{-1 / 2}|0\rangle=0
$$

a result that confirms the fermionic nature of the $b$ modes. Setting $m=-2$ and $n \geq 3$, we find that

$$
b_{-3 / 2+n} b_{-1 / 2}|0\rangle=-b_{-1 / 2} b_{-3 / 2+n}|0\rangle+\left\{b_{-3 / 2+n}, b_{-1 / 2}\right\}|0\rangle=0
$$

The anticommutator $\left\{b_{-3 / 2+n}, b_{-1 / 2}\right\}$ must then vanish when $n \geq 3$. But since the result of this anticommutator should depend only upon the sum of the indices, we conclude that for all values of $p$ and $r$ such that $p+r>0,\left\{b_{p}, b_{r}\right\}=0$. By symmetry this must also hold when $p+r<0$. Consider finally the case where $m=1$ and $n=-1$, which yields

$$
b_{1 / 2} b_{-1 / 2}|0\rangle=-b_{-1 / 2} b_{1 / 2}|0\rangle+\left\{b_{1 / 2}, b_{-1 / 2}\right\}|0\rangle=\left[L_{0}+\left.\frac{1}{2} n(n-1)\right|_{n=-1}\right]|0\rangle=|0\rangle
$$

Therefore the anticommutator $\left\{b_{p}, b_{r}\right\}$ is 1 when $p+r=0$. We have thus recovered the standard anticommutation relation of the free fermion:

$$
\left\{b_{p}, b_{r}\right\}=\delta_{p+r, 0}
$$

However, (2.10) contains much more information than the mere specification of (C.8). In order to extract it, let us return to the case where both sides of the equation act on a generic state $\left|\phi_{q}\right\rangle$. By treating separately the two sectors $q=0,1$, and by reorganizing the sum appropriately, we find that

$$
L_{p}=\sum_{\ell \in \mathbf{Z}+\epsilon}(\ell+1 / 2) b_{p-\ell} b_{\ell} \quad(p \neq 0)
$$

(where $\epsilon$ is $1 / 2$ for NS and 0 for $\mathrm{R}$ ) and

$$
L_{0}=\sum_{\ell \in \mathbf{Z}+\epsilon>0} \ell: b_{p-\ell} b_{\ell}:+\frac{1}{16} \delta_{R}
$$

where $\delta_{R}$ is $0(1)$ in the NS (R) sector. These are the expected relations. 
Consider now the structure of the singular vectors. In the free-fermion reinterpretation, these simply turn into highest-weight conditions. For instance, for the vacuum vector, we have:

$$
\begin{aligned}
& \mathcal{B}_{0}|0\rangle=b_{1 / 2}|0\rangle=0 \\
& \mathcal{A}_{-1}^{3}|0\rangle=b_{3 / 2} b_{1 / 2} b_{-1 / 2}|0\rangle=-b_{3 / 2} b_{-1 / 2} b_{1 / 2}|0\rangle+b_{3 / 2}|0\rangle=0
\end{aligned}
$$

Therefore, there are no singular vectors in the fermionic Fock space and the counting of states becomes substantially simplified. Moreover, since $\mathcal{A}=\mathcal{B}$, we do not have to consider separately $\mathcal{A}$ and $\mathcal{B}$ strings of states. Using the $\mathcal{A}$-string description, we know, from lemma 1 , that the states can be written in the form

$$
b_{-n_{1}} b_{-n_{2}} \cdots b_{-n_{j}}\left|\phi_{q}\right\rangle
$$

with the $n_{i}$ non-decreasing from right to left and all $n_{i}>0$. But given the fermionic nature of the $b$ modes, two adjacent $n_{i}$ cannot be equal, so that we actually have an increasing sequence of integers. The number of states at a given level $s$ is thus determined by the number of partitions of $s$ into distinct parts. The states can further be separated according to their statistics, that is, their fermionic or bosonic nature. In this way, we recover the characters presented in (1.1).

\section{Acknowledgment}

We would like to thank Y. Saint-Aubin for useful and stimulating discussions, B. McCoy for clarifying remarks and for pointing out [14]) to us and L. Bégin for his help in verifying the character formulae by computer. We also thank J.-F. Carrier and B. Paquet for exploratory calculations related to this work.

\section{REFERENCES}

1. B.M. McCoy and J.H.H. Perk, Nucl. Phys. B285 [FS19] (1987) 279.

2. A.B. Zamolodchikov and V.A. Fateev. Sov. Phys. JETP 82 (1985) 215.

3. A.B. Zamolodchikov and V.A. Fateev, Sov. Phys. JETP 63 (1986) 913.

4. A.B. Zamolodchikov and V.A. Fateev. Theor. Math. Phys. 71 (1987) 163.

5. D. Nemeschansky, Nucl. Phys. B363 (1989) 665.

6. D. Nemeschansky, Phys. Lett B224 (1989) 121.

7. J. Distler and Z. Qiu, Nucl.Phys. B336 (1990) 533.

8. D. Gepner and Z. Qiu, Nucl. Phys. B285 [FS19] (1987) 423. 
9. V.A. Fateev, Int J. Mod Phys. A6 (1991) 2109.

10. P. Di Francesco, P. Mathieu and D. Sénéchal, Conformal Field theory, Springer Verlag, 1997.

11. R. Dijkgraaf, C. Vafa, E. Verlinde and H. Verlinde, Comm. Math. Phys. 123 (1989) 485.

12. A. Rocha-Caridi, in Vertex Operators in Mathematics and Physics, ed. by J. Lepowsky et al, Mathematical Sciences Research Institute Publications vol. 3, Springer-Verlag, New-York (1985), 451.

13. V. Kac and D. Peterson, Adv. Math. 53 (1984) 125

14. J. Lepowsky and M. Primc, Contemporary Mathematics 46 AMS, Providence, 1985.

15. L. Lepowsky and R.L. Wilson, Invent. Math. 77 (1984) 199.

16. R. Kedem, T.R. Klassen, B. M. McCoy and E. Melzer, Phys. Lett. B304 (1993) 263.

17. P. Di Francesco and P. Mathieu, Phys. Lett. B278 (1992) 79; P. Mathieu and G. Watts, Nucl. Phys. B475 (1996) 361. 